\title{
Uncontrolled sepsis: a systematic review of translational immunology studies in intensive care medicine
}

David J Cain, Ana Gutierrez del Arroyo and Gareth L Ackland

*Correspondence: g.ackland@ucl.ac.uk Clinical Physiology, Wolfson Institute for Biomedical Research, Department of Medicine, University College London, London WC1E 6BT, UK

\begin{abstract}
Background: The design of clinical immunology studies in sepsis presents several fundamental challenges to improving the translational understanding of pathologic mechanisms. We undertook a systematic review of bed-to-benchside studies to test the hypothesis that variable clinical design methodologies used to investigate immunologic function in sepsis contribute to apparently conflicting laboratory data, and identify potential alternatives that overcome various obstacles to improve experimental design.
\end{abstract}

Methods: We performed a systematic review of the design methodology employed to study neutrophil function (respiratory burst), monocyte endotoxin tolerance and lymphocyte apoptosis in the intensive care setting, over the past 15 years. We specifically focussed on how control samples were defined, taking into account age, gender, ethnicity, concomitant therapies, timing of sample collection and the criteria used to diagnose sepsis.

Results: We identified 57 eligible studies, the majority of which (74\%) used case-control methodology. Healthy volunteers represented the control population selected in $83 \%$ of studies. Comprehensive demographic data on age, gender and ethnicity were provided in $\leq 48 \%$ of case control studies. Documentation of diseases associated with immunosuppression, malignancy and immunomodulatory therapies was rare. Less than half (44\%) of studies undertook independent adjudication for the diagnosis of sepsis while $68 \%$ provided microbiological data. The timing of sample collection was defined by highly variable clinical criteria. By contrast, surgical studies avoided many such confounders, although only one study in surgical patients monitored the study group for development of sepsis.

Conclusions: We found several important and common limitations in the clinical design of translational immunologic studies in human sepsis. Major elective surgery overcame many of these methodological limitations. The failure of adequate clinical design in mechanistic studies may contribute to the lack of translational therapeutic progress in intensive care medicine.

Keywords: Sepsis; Immunology; Human; Critical care; Surgical intensive care

\section{Springer}

(c) 2014 Cain et al.; licensee Springer. This is an Open Access article distributed under the terms of the Creative Commons Attribution License (http://creativecommons.org/licenses/by/2.0), which permits unrestricted use, distribution, and reproduction in any medium, provided the original work is properly credited. 


\section{Background}

Mortality from sepsis is persistently high, and may even be rising despite decades of research [1,2]. Promising pre-clinical immunomodulatory therapies have failed in clinical practice [3-5] perhaps attributable, in part, to differences between human and rodent immunology [6]. However, an alternative explanation is that the heterogeneous etiology, presentation and progression of human sepsis generate confounding factors that distort the interpretation of clinical immunologic studies. Thus, the identification of appropriate controls, diagnostic accuracy, demographic influences and therapies with immunomodulatory off-target effects are critical considerations in interpreting translational work.

We therefore systematically reviewed the clinical experimental design of studies in three key areas of bed-to-benchside immunologic research in sepsis, focusing in particular on comparator groups and the documentation of known confounding factors. We also explored how the investigation of immune mechanisms in other clinical scenarios - trauma and major elective surgery - associated with the development of sepsis may help refine experimental design.

\section{Methods}

A Pubmed search was performed for the terms 'Neutrophil respiratory burst' OR 'Monocyte endotoxin tolerance' OR 'Lymphocyte apoptosis' AND 'Sepsis' OR 'Trauma' OR 'Surgery', restricted to adult human studies published between 03 January 1998 and 03 January 2013. The abstract of each paper was manually assessed for suitability. In vitro studies of healthy volunteer cells were excluded.

\section{Clinical demographics}

For all eligible manuscripts, we recorded the primary author, year of publication and clinical setting. The number, age, gender, clinical severity score of subjects and their corresponding controls, in whom the same assay of immune function was performed, were compared. The criteria used to define sepsis - complete with evidence for microbiological confirmation and independent adjudication of the sepsis diagnosis - were also recorded. Since immune cell effector function may change over the course of sepsis, we also recorded details of the timing of initial and subsequent blood samples, and the reason for blood sampling itself. Given that a recent report detected differences in genomic markers of inflammation that associate with survival within the first $24 \mathrm{~h}$ of intensive care admission [7], we assessed whether samples were obtained within, or beyond, this 24-h window. Since several commonly used therapies used in intensive care medicine exhibit immune modulating effects, we also recorded whether common immunomodulatory agents including antibiotics [8], glucocorticoids [9] and sedative agents [10] were documented. Reporting of pre-existing immunosuppressive or malignant disease - or their specific exclusion - was also recorded.

\section{Study aims}

The specific aims of each study were recorded with regard to the experimental context and primary conclusion. The context within which each of the three functional assays was studied was classified as: Pathophysiological - observational mechanistic studies detailing evolution of the assay response in clinical samples; Experimental - use of patient samples for more detailed experimental investigations beyond the assay itself; 
Clinical outcome - correlation of outcome measure with assay response; Biomarker comparison - correlation of alternative assay with functional assay.

\section{Laboratory samples}

We recorded whether an a priori power analysis had been performed to determine the number of subjects/controls needed to refute the primary hypothesis. Sample timing and key aspects of experimental technique were compared between sepsis and control subjects. Associations made between immune cell function and clinical outcome were noted.

\section{Statistics}

Data are presented as mean \pm SD, or median (interquartile range). Age data in primary studies was used to construct $95 \%$ confidence intervals in order to assess whether differences existed between control and study populations (NCSS 8, Kaysville, UT, USA).

\section{Results}

Fifty-seven eligible studies were identified, as summarised in Figure 1. Data is displayed into 3 tables for each immune assay, titled "Principal features of studies" (Tables 1, 2 and 3), "Demographic information" (Tables 4, 5 and 6) and "Experimental conduct and exclusion criteria" (Tables 7, 8 and 9).

\section{Source of experimental control subjects}

No studies reported a priori power analyses based on either preceding laboratory data or ex vivo clinical research. The majority of studies $(42 / 57 ; 74 \%)$ used case-control

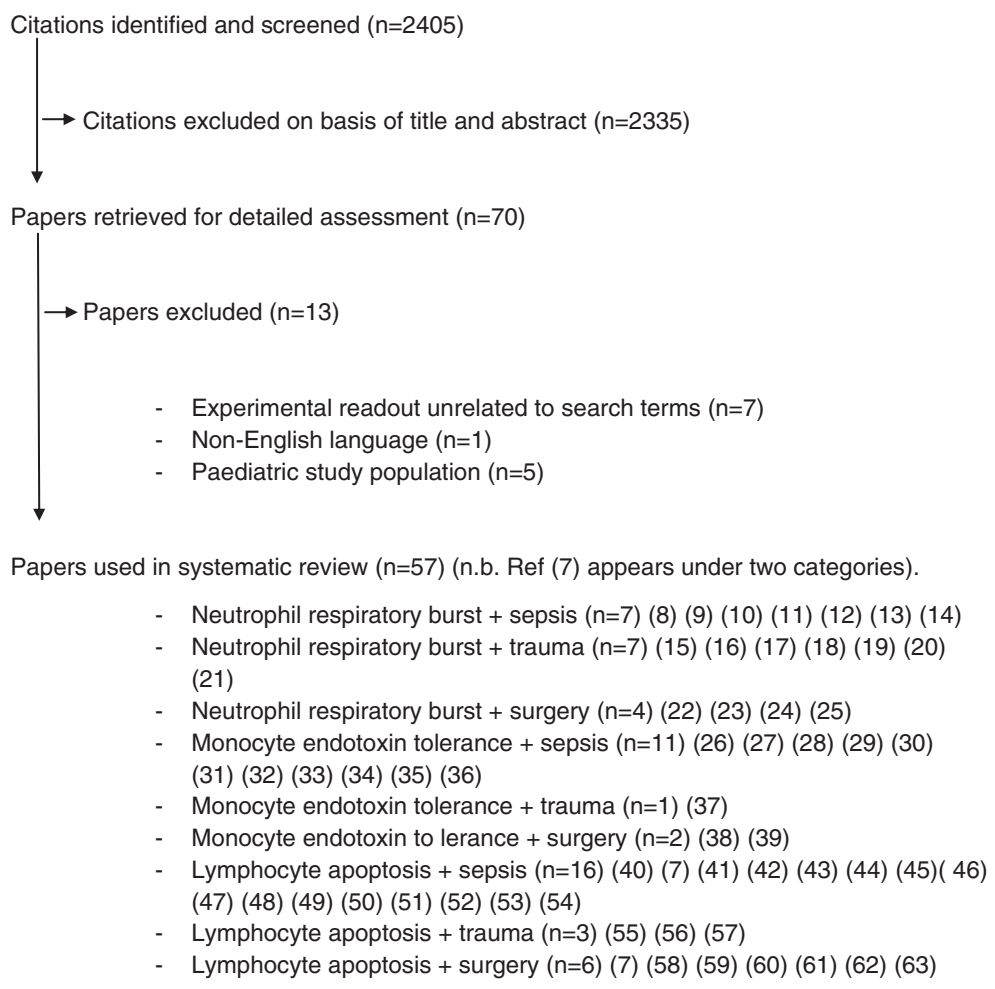

Figure 1 Flow diagram illustrating study identification and inclusion [11-66]. 
Table 1 Principal features of neutrophil respiratory burst studies

\begin{tabular}{|c|c|c|c|c|c|c|}
\hline Author & $\begin{array}{l}\text { Study } \\
\text { population }\end{array}$ & $\begin{array}{l}\text { Subjects } \\
(n)\end{array}$ & $\begin{array}{l}\text { Control } \\
\text { population }\end{array}$ & $\begin{array}{l}\text { Control } \\
(n)\end{array}$ & $\begin{array}{l}\text { Experimental } \\
\text { context }\end{array}$ & $\begin{array}{l}\text { Outcome measure } \\
\text { correlated with } \\
\text { immune readout }\end{array}$ \\
\hline Santos [12] & Sepsis & 49 & $\begin{array}{l}\text { Healthy } \\
\text { volunteer }\end{array}$ & 19 & $\begin{array}{l}\text { Clinical } \\
\text { outcome }\end{array}$ & Yes \\
\hline $\begin{array}{l}\text { Paunel- } \\
\text { Gorgulu [19] }\end{array}$ & Trauma & 7 & $\begin{array}{l}\text { Healthy } \\
\text { volunteer }\end{array}$ & 6 & Experimental & No \\
\hline Bruns [13] & $\begin{array}{l}\text { Sepsis } \\
\text { (cirrhotics) }\end{array}$ & 45 & $\begin{array}{l}\text { Healthy } \\
\text { volunteer } \\
\text { and cohort }\end{array}$ & 9 and 39 & Pathophysiological & No \\
\hline Shih [20] & Trauma & 32 & $\begin{array}{l}\text { Healthy } \\
\text { volunteer }\end{array}$ & $\begin{array}{l}\text { Not } \\
\text { provided }\end{array}$ & $\begin{array}{l}\text { Biomarker } \\
\text { comparison }\end{array}$ & Yes \\
\hline Kasten [21] & Trauma & 3 & $\begin{array}{l}\text { Healthy } \\
\text { volunteer }\end{array}$ & 3 & Pathophysiological & No \\
\hline Valente [22] & Trauma & 24 & $\begin{array}{l}\text { Healthy } \\
\text { volunteer }\end{array}$ & 11 & Pathophysiological & No \\
\hline Kawasaki [26] & $\begin{array}{l}\text { Elective } \\
\text { surgery }\end{array}$ & $\begin{array}{l}20 \\
(10,10)\end{array}$ & Cohort & 20 & Pathophysiological & No \\
\hline Frohlich [27] & $\begin{array}{l}\text { Elective } \\
\text { surgery }\end{array}$ & 20 & Cohort & 20 & Experimental & No \\
\hline Martins [14] & Sepsis & 16 & $\begin{array}{l}\text { Healthy } \\
\text { volunteer }\end{array}$ & 16 & Pathophysiological & Yes \\
\hline Barth [15] & Sepsis & 27 & $\begin{array}{l}\text { Healthy } \\
\text { volunteer }\end{array}$ & 11 & $\begin{array}{l}\text { Biomarker } \\
\text { comparison }\end{array}$ & No \\
\hline Mariano [16] & $\begin{array}{l}\text { Sepsis } \\
\text { (renal } \\
\text { replacement } \\
\text { therapy) }\end{array}$ & 7 & $\begin{array}{l}\text { Haemodialysis } \\
\text { patients }\end{array}$ & 10 & Pathophysiological & No \\
\hline Quaid [23] & Trauma & 7 & $\begin{array}{l}\text { Healthy } \\
\text { volunteer }\end{array}$ & $\begin{array}{l}\text { Not } \\
\text { provided }\end{array}$ & Pathophysiological & No \\
\hline Wiezer [28] & $\begin{array}{l}\text { Elective } \\
\text { surgery }\end{array}$ & $\begin{array}{l}22 \\
(6,6,10)\end{array}$ & Cohort & 22 & $\begin{array}{l}\text { Pathophysiological/ } \\
\text { experimental }\end{array}$ & Yes \\
\hline Ahmed [17] & Sepsis & 32 & $\begin{array}{l}\text { Healthy } \\
\text { volunteer }\end{array}$ & 17 & Pathophysiological & No \\
\hline Shih [29] & $\begin{array}{l}\text { Trauma/ } \\
\text { surgery }\end{array}$ & 18 & $\begin{array}{l}\text { Cohort } \\
\text { and healthy } \\
\text { volunteer }\end{array}$ & 18 & Pathophysiological & No \\
\hline Ertel [24] & Trauma & $10(5,5)$ & $\begin{array}{l}\text { Elective } \\
\text { surgery }\end{array}$ & 10 & Pathophysiological & No \\
\hline Ogura [25] & Trauma & $\begin{array}{l}24 \\
\text { (7 infected) }\end{array}$ & $\begin{array}{l}\text { Cohort and } \\
\text { healthy } \\
\text { volunteer }\end{array}$ & $\begin{array}{l}24 \text { and } \\
15\end{array}$ & Pathophysiological & Yes \\
\hline Pascual [18] & Sepsis & 23 & $\begin{array}{l}\text { Elective } \\
\text { surgery }\end{array}$ & 23 & $\begin{array}{l}\text { Pathophysiological/ } \\
\text { experimental }\end{array}$ & No \\
\hline
\end{tabular}

Subjects: values within brackets refer to subgroups within the study.

methodology. Control samples were obtained from healthy volunteers in $(35 / 42 ; 83 \%)$, with the remainder using a variety of loosely defined clinical phenotypes (Figure 2, Tables 1, 2 and 3). The exception was elective surgical patients, where preoperative samples served as appropriate controls. Cohort methodology, where samples including controls were obtained serially from the same patient, was employed in 14/57 (25\%) of studies. The majority of cohort studies were conducted in elective surgical patients $(12 / 14 ; 86 \%)$. 
Table 2 Principal features of monocyte tolerance studies

\begin{tabular}{|c|c|c|c|c|c|c|}
\hline Author & $\begin{array}{l}\text { Study } \\
\text { population }\end{array}$ & $\begin{array}{l}\text { Subjects } \\
(n)\end{array}$ & $\begin{array}{l}\text { Control } \\
\text { population }\end{array}$ & $\begin{array}{l}\text { Controls } \\
(n)\end{array}$ & $\begin{array}{l}\text { Experimental } \\
\text { context }\end{array}$ & $\begin{array}{l}\text { Outcome measure } \\
\text { correlated with } \\
\text { immune readout }\end{array}$ \\
\hline Liu [30] & Sepsis & 2 & $\begin{array}{l}\text { Healthy } \\
\text { volunteer }\end{array}$ & 2 & Experimental & No \\
\hline $\begin{array}{l}\text { Buttenschoen } \\
\text { [41] }\end{array}$ & $\begin{array}{l}\text { Elective } \\
\text { surgery }\end{array}$ & 20 & Cohort & 20 & Pathophysiological & No \\
\hline Pachot [31] & Sepsis & 47 & $\begin{array}{l}\text { Healthy } \\
\text { volunteer }\end{array}$ & 21 & Pathophysiological & Yes \\
\hline West [32] & Sepsis & 7 & $\begin{array}{l}\text { Healthy } \\
\text { volunteer, } \\
\text { elective } \\
\text { surgery } \\
\text { and SIRS }\end{array}$ & $\begin{array}{l}16,5 \\
\text { and } 4\end{array}$ & Pathophysiological & No \\
\hline Harter [33] & Sepsis & 21 & $\begin{array}{l}\text { Healthy } \\
\text { volunteer }\end{array}$ & 12 & Pathophysiological & No \\
\hline Flohe [40] & $\begin{array}{l}\text { Surgery } \\
\text { in trauma } \\
\text { patients }\end{array}$ & 16 & $\begin{array}{l}\text { Healthy } \\
\text { volunteer }\end{array}$ & 12 & Pathophysiological & No \\
\hline Escoll [34] & Sepsis & $3(5)$ & $\begin{array}{l}\text { Healthy } \\
\text { volunteer }\end{array}$ & 3 & Pathophysiological & No \\
\hline Heagy [39] & $\begin{array}{l}\text { ICU } \\
\text { patients } \\
\text { (sepsis) }\end{array}$ & 62 & $\begin{array}{l}\text { Healthy } \\
\text { volunteer }\end{array}$ & 15 & Clinical outcome & Yes \\
\hline Calvano [35] & Sepsis & $18(10)$ & $\begin{array}{l}\text { Healthy } \\
\text { volunteer }\end{array}$ & $15(6)$ & Pathophysiological & No \\
\hline Sfeir [36] & Sepsis & 10 & $\begin{array}{l}\text { Healthy } \\
\text { volunteer }\end{array}$ & 10 & Pathophysiological & No \\
\hline Kawasaki [42] & $\begin{array}{l}\text { Elective } \\
\text { surgery }\end{array}$ & 20 & Cohort & 20 & Pathophysiological & No \\
\hline Heagy [37] & Sepsis & 58 & $\begin{array}{l}\text { Healthy } \\
\text { volunteer }\end{array}$ & 14 & Clinical outcome & Yes \\
\hline Bergmann [38] & Sepsis & $30(2)$ & $\begin{array}{l}\text { Healthy } \\
\text { volunteer }\end{array}$ & 12 & Pathophysiological & No \\
\hline
\end{tabular}

Subjects/controls: numbers in brackets refer to subgroups within study.

Age, gender and ethnicity

Advanced age is associated with progressively impaired innate and adaptive immunity [67]. Less than half of case control studies $(20 / 42 ; 48 \%)$ reported the age distribution of both study and control populations. In studies where age was reported, the critically ill patients studied were often older than the control population. Female gender is associated with improved clinical outcomes following sepsis $[68,69]$ and increased longevity compared to males in general. Information on gender was provided in $(26 / 42 ; 62 \%)$ of case-control studies. Significant variation in the incidence of sepsis has been reported according to ethnicity [70], which may reflect residual confounding or plausible biologic differences in susceptibility. However, only one study reported the ethnicity of patients.

\section{Co-morbidity}

Various comorbidities ranging from cardiac failure to active malignancy are associated with important deleterious alteration in effective immune function, independent of those described in sepsis $[71,72]$. The majority of studies $(34 / 57 ; 60 \%)$ excluded patients with overt immunosuppression while a minority $(8 / 57 ; 14 \%)$ excluded those with malignancy (Figure 3). 
Table 3 Principal features of lymphocyte apoptosis studies

\begin{tabular}{|c|c|c|c|c|c|c|}
\hline Author & $\begin{array}{l}\text { Study } \\
\text { population }\end{array}$ & $\begin{array}{l}\text { Subjects } \\
(n)\end{array}$ & $\begin{array}{l}\text { Control } \\
\text { population }\end{array}$ & $\begin{array}{l}\text { Controls } \\
(n)\end{array}$ & $\begin{array}{l}\text { Experimental } \\
\text { context }\end{array}$ & $\begin{array}{l}\text { Outcome } \\
\text { measure } \\
\text { correlated } \\
\text { with } \\
\text { immune } \\
\text { readout }\end{array}$ \\
\hline Roger [43] & Sepsis & 48 & $\begin{array}{l}\text { Healthy } \\
\text { volunteer }\end{array}$ & 15 & Pathophysiological & No \\
\hline $\begin{array}{l}\text { Bandyopadhyay } \\
\text { [58] }\end{array}$ & Trauma & 113 & $\begin{array}{l}\text { Healthy } \\
\text { volunteer }\end{array}$ & $?$ & Pathophysiological & No \\
\hline White [11] & Sepsis & 60 & $\begin{array}{l}\text { Gram } \\
\text { negative } \\
\text { infection and } \\
\text { healthy } \\
\text { volunteer }\end{array}$ & 15 and 20 & Pathophysiological & Yes \\
\hline White [11] & $\begin{array}{l}\text { Elective } \\
\text { surgery } \\
\text { (infective } \\
\text { complications) }\end{array}$ & 19 & Cohort & 41 & $"$ & $"$ \\
\hline Zhang [44] & Sepsis & 19 & $\begin{array}{l}\text { Healthy } \\
\text { volunteer }\end{array}$ & 22 & Pathophysiological & No \\
\hline Guignant [45] & Sepsis & 64 & $\begin{array}{l}\text { Healthy } \\
\text { volunteer }\end{array}$ & 49 & Pathophysiological & No \\
\hline Vaki [46] & Sepsis & $48(68)$ & $\begin{array}{l}\text { Healthy } \\
\text { volunteer }\end{array}$ & 20 & Pathophysiological & No \\
\hline Slotwinski [62] & $\begin{array}{l}\text { Elective } \\
\text { surgery }\end{array}$ & $\begin{array}{l}50 \\
(26,24)\end{array}$ & Cohort & 50 & $\begin{array}{l}\text { Experimental/ } \\
\text { clinical outcome }\end{array}$ & No \\
\hline Gogos [47] & Sepsis & $\begin{array}{l}\text { PN 183, } \\
\text { CAP 97, IA } \\
100, \text { PB 61, } \\
\text { HAP } 64\end{array}$ & N/A & & Pathophysiological & Yes \\
\hline Hoogerwerf [48] & Sepsis & 16 & $\begin{array}{l}\text { Healthy } \\
\text { volunteer }\end{array}$ & 24 & Pathophysiological & No \\
\hline Yousef [49] & Sepsis & 32 & $\begin{array}{l}\text { SIRS and } \\
\text { without SIRS }\end{array}$ & $35 / 33$ & Patient outcome & Yes \\
\hline Turrel-Davin [50] & Sepsis & 13 & $\begin{array}{l}\text { Healthy } \\
\text { volunteer }\end{array}$ & 15 & $\begin{array}{l}\text { Biomarker } \\
\text { comparison }\end{array}$ & No \\
\hline Pelekanou [51] & Sepsis & VAP 36 & $\begin{array}{l}\text { Other } \\
\text { infections }\end{array}$ & 32 & Pathophysiology & No \\
\hline Papadima [61] & $\begin{array}{l}\text { Elective } \\
\text { surgery }\end{array}$ & $\begin{array}{l}40 \\
(21,19)\end{array}$ & Cohort & 40 & Pathophysiological & No \\
\hline Delogu [52] & Sepsis & 16 & ? 'individuals' & & Pathophysiological & No \\
\hline Weber [53] & Sepsis & 16 & $\begin{array}{l}\text { Non-infected } \\
\text { ICU and } \\
\text { healthy } \\
\text { volunteer }\end{array}$ & 10 and 11 & Pathophysiological & No \\
\hline Roth [54] & Sepsis & 15 & $\begin{array}{l}\text { Healthy } \\
\text { volunteer }\end{array}$ & 20 & Pathophysiological & No \\
\hline Le Tulzo [55] & Sepsis & $\begin{array}{l}47 \\
(25,23)\end{array}$ & $\begin{array}{l}\text { SIRS and } \\
\text { healthy } \\
\text { volunteer }\end{array}$ & 7 and 25 & $\begin{array}{l}\text { Pathophysiological/ } \\
\text { clinical outcome }\end{array}$ & Yes \\
\hline Hotchkiss [56] & Sepsis & $\begin{array}{l}27 \text { (FC 5) } \\
\text { (3 intraop, } \\
24 \text { autopsy) }\end{array}$ & $\begin{array}{l}\text { Critically ill } \\
\text { non-septic } \\
\text { and trauma }\end{array}$ & $\begin{array}{l}16 \text { and } 25 \\
\text { (FC 6) (3 } \\
\text { prospective, } \\
13 \\
\text { retrospective) }\end{array}$ & Pathophysiological & No \\
\hline Delogu [63] & $\begin{array}{l}\text { Elective } \\
\text { surgery }\end{array}$ & 18 & Cohort & 18 & Pathophysiological & No \\
\hline
\end{tabular}


Table 3 Principal features of lymphocyte apoptosis studies (Continued)

\begin{tabular}{|c|c|c|c|c|c|c|}
\hline Pellegrini [59] & Trauma & $\begin{array}{l}17 \\
\text { (+13 burns) }\end{array}$ & $\begin{array}{l}\text { Healthy } \\
\text { volunteer }\end{array}$ & 17 & $\begin{array}{l}\text { Clinical outcome/ } \\
\text { pathophysiological }\end{array}$ & $\begin{array}{l}\text { (Correlate } \\
\text { to MODS) }\end{array}$ \\
\hline Delogu [64] & Surgical & 15 & $\begin{array}{l}\text { Healthy } \\
\text { volunteer }\end{array}$ & 10 & $\begin{array}{l}\text { Pathophysiological/ } \\
\text { patient outcome }\end{array}$ & Yes \\
\hline Hotchkiss [60] & Trauma & 10 & $\begin{array}{l}\text { Elective } \\
\text { surgery }\end{array}$ & $\begin{array}{l}6 \text { (all } \\
\text { prospective) }\end{array}$ & Pathophysiological & No \\
\hline Hotchkiss [57] & Sepsis & 20 & $\begin{array}{l}\text { Non septic } \\
\text { prospective/ } \\
\text { non-septic } \\
\text { retrospective/ } \\
\text { prospective } \\
\text { trauma } \\
\text { splenectomy/ } \\
\text { prospective } \\
\text { colectomy/ } \\
\text { retrospective } \\
\text { colectomy }\end{array}$ & $1 / 9 / 6 / 2 / 8$ & Pathophysiological & No \\
\hline Sasajima [65] & $\begin{array}{l}\text { Elective } \\
\text { surgery }\end{array}$ & $\begin{array}{l}16 \\
(11,5)\end{array}$ & Cohort & 16 & Pathophysiological & No \\
\hline Sugimoto [66] & $\begin{array}{l}\text { Elective } \\
\text { surgery }\end{array}$ & $10(5,5)$ & Cohort & 10 & Pathophysiological & No \\
\hline
\end{tabular}

Clinical definition of sepsis

A high proportion of studies (26/33; 79\%) defined sepsis in accordance with the ACCP/ SCCM [73,74] or Surviving Sepsis Campaign (2008 update) [75] criteria. Of those studies which used standard consensus conference criteria, $(15 / 26,58 \%)$ included patients with 'sepsis', (20/26; 77\%) included those with 'severe sepsis' and (24/26, 92\%) included those with 'septic shock'. In a large minority of these 26 studies $(11 / 26 ; 42 \%)$, subcategories defining sepsis were not compared separately, but combined. Immunologic studies in trauma and surgical patient samples usually did not document $(18 / 24 ; 75 \%)$ whether patients developed an infection during the course of the study. In these studies, the majority (5/6) used established consensus conference criteria.

\section{Microbiological definitions of sepsis}

Independent adjudication of the definition of sepsis used in studies was undertaken in $17 / 57(30 \%)$ of studies. Since recent basic laboratory studies have demonstrated that the clinical signs/symptoms of sepsis are frequently mimicked by non-pathogenic molecules [76,77], we sought to establish whether microbial evidence for sepsis was presented. Microbiological data were provided in 25/57 (44\%).

\section{Severity of critical illness}

A minority of studies $(19 / 57 ; 33 \%)$ provided data on organ dysfunction related to sepsis severity, such as APACHE-II or SAPS II. When a severity index was used, a wide range was reported within individual studies suggesting substantial heterogeneity. In studies where mortality was reported $(4 / 57 ; 7 \%)$, severity of critical illness was not reported in those patients who survived.

\section{Timing of experimental samples}

The timing of the index blood sample obtained from septic patients was described in the majority $(26 / 33 ; 79 \%)$ of cases. However, the criteria for initial sampling were not 
Table 4 Demographic information of neutrophil respiratory burst studies

\begin{tabular}{|c|c|c|c|c|c|c|c|c|c|c|c|c|c|}
\hline \multirow[t]{2}{*}{ Author } & \multicolumn{3}{|l|}{ Age } & \multicolumn{3}{|c|}{ Gender (\%male) } & \multirow{2}{*}{$\begin{array}{l}\text { Subject } \\
\text { ethnicity } \\
\text { detailed }\end{array}$} & \multicolumn{3}{|c|}{ Severity of subject disease } & \multicolumn{3}{|c|}{ Subject drug exposure documentation } \\
\hline & Subjects & Controls & $\begin{array}{l}\text { Statistical } \\
\text { test result }\end{array}$ & Subjects & Controls & $\begin{array}{l}\text { Statistical } \\
\text { test result }\end{array}$ & & Index & Score & No. Groups & Sedatives & Antibiotics & Steroids \\
\hline Santos [12] & $60 \pm 17$ & $55.3 \pm 18$ & $\mathrm{~N}$ & 57 & 53 & $\mathrm{~N}$ & $\mathrm{~N}$ & APACHE II & $17(4$ to 30$)$ & 3 & $\mathrm{~N}$ & $\mathrm{~N}$ & $\mathrm{~N}$ \\
\hline Gorgulu [19] & $46 \pm 4$ & $33 \pm 2$ & $\mathrm{~N}^{*}(p<0.001)$ & 74 & 59 & $\mathrm{~N}$ & $\mathrm{~N}$ & Mortality & $9 \%$ & 1 & $\mathrm{~N}$ & $\mathrm{~N}$ & $\mathrm{~N}$ \\
\hline Bruns [13] & 58 (40 to 80$)$ & 45 (37 to 82$) ; 58$ (?) & 0.437 & 82 & $73 / 48$ & 0.341 & $\mathrm{~N}$ & - & & 1 & $\mathrm{~N}$ & $\mathrm{~N}$ & $\mathrm{~N}$ \\
\hline Shih [20] & $33 \pm 14$ & $?$ & $\mathrm{~N}$ & 66 & $?$ & $\mathrm{~N}$ & $\mathrm{~N}$ & ISS & 23 & 2 & $\mathrm{~N}$ & $\mathrm{~N}$ & $\mathrm{~N}$ \\
\hline Kasten [21] & $36 \pm 2$ & $38 \pm 2$ & $p>0.05$ & 100 & 100 & $p>0.05$ & $\mathrm{~N}$ & ISS & 23 & 1 & $\mathrm{~N}$ & $\mathrm{~N}$ & $\mathrm{~N}$ \\
\hline Valente [22] & 75 & $>65$ & $\mathrm{~N}$ & 46 & $?$ & $\mathrm{~N}$ & $\mathrm{~N}$ & ISS & 15.00 & 1 & $\mathrm{~N}$ & $\mathrm{~N}$ & $\mathrm{~N}$ \\
\hline Kawasaki [26] & $52 \pm 4 ; 54 \pm 4$ & N/A & $\mathrm{N}$ & 70 & 70 & $p>0.05$ & $\mathrm{~N}$ & ASA & | to || & 2 & Y & $\mathrm{N}$ & $N$ \\
\hline Frohlich [27] & $66 \pm 10 ; 69 \pm 6$ & $\mathrm{~N} / \mathrm{A}$ & $\mathrm{N}$ & 40 & 20 & $\mathrm{~N}$ & $\mathrm{~N}$ & ASA & 1 & 2 & $Y t$ & $Y t$ & $Y t$ \\
\hline Martins [14] & $50 \pm 21$ & $31 \pm 6$ & $N^{*}(p=0.0011)$ & $?$ & $?$ & $\mathrm{~N}$ & N & Mortality & $38 \%$ & 2 & $\mathrm{~N}$ & $\mathrm{~N}$ & $\mathrm{~N}$ \\
\hline Barth [15] & $\mathrm{N} / \mathrm{S}$ (36 to 82 ) & 24 (22 to 50$)$ & $\mathrm{N}$ & 60 & 36 & $\mathrm{~N}$ & $\mathrm{~N}$ & Mortality & $37 \%$ & 1 & $\mathrm{~N}$ & $\mathrm{~N}$ & $\mathrm{~N}$ \\
\hline Mariano [16] & $67 \pm 4$ & $?$ & $\mathrm{~N}$ & $?$ & $?$ & $\mathrm{~N}$ & $\mathrm{~N}$ & - & & 1 & $\mathrm{~N}$ & $\mathrm{~N}$ & $N$ \\
\hline Quaid [23] & 37 (20 to 71$)$ & $?$ & $\mathrm{~N}$ & $?$ & $?$ & $\mathrm{~N}$ & $\mathrm{~N}$ & ISS & 24 (17 to 34$)$ & & $\mathrm{N}$ & $\mathrm{N}$ & $\mathrm{N}$ \\
\hline Wiezer [28] & $\begin{array}{l}57 \pm 3 ; 62 \pm 2 \\
58 \pm 5\end{array}$ & $?$ & $\mathrm{~N}$ & $83,66,70$ & & N & $N$ & APACHE III & $\begin{array}{l}\text { Graphs } \\
\text { (no difference) }\end{array}$ & 3 & $\mathrm{~N}$ & $\mathrm{~N}$ & $\mathrm{~N}$ \\
\hline Ahmed [17] & $55 \pm 6$ & $36 \pm 16$ & $N^{*}(p<0.0001)$ & 46 & $?$ & $\mathrm{~N}$ & $N$ & APACHE ॥ & $20 \pm 1$ & 1 & N & $\mathrm{N}$ & $\mathrm{N}$ \\
\hline Shih [29] & $42 \pm 19$ & $N / S$ & $\mathrm{~N}$ & 55 & $?$ & $\mathrm{~N}$ & $\mathrm{~N}$ & ISS & $26 \pm 7.2$ & 3 & $\mathrm{~N}$ & $\mathrm{~N}$ & $\mathrm{~N}$ \\
\hline Ertel [24] & $\mathrm{N} / \mathrm{S}$ & $?$ & $\mathrm{~N}$ & $?$ & $?$ & $\mathrm{~N}$ & $\mathrm{~N}$ & AIS & $\begin{array}{l}\text { Head } 4.5 \pm 0.2 \\
\text { Chest } 4.1 \pm 0.1\end{array}$ & 1 & $\mathrm{~N}$ & $\mathrm{~N}$ & $N$ \\
\hline Ogura [25] & $40 \pm 19$ & $35 \pm 6$ & $\mathrm{~N}$ & 75 & $?$ & $\mathrm{~N}$ & $\mathrm{~N}$ & ISS & $31 \pm 10$ & 2 & N & $\mathrm{N}$ & $\mathrm{N}$ \\
\hline Pascual [18] & 59 (27 to 81 ) & 45 (27 to 81 ) & $p>0.05$ & 51 & 43 & N & $\mathrm{N}$ & Mortality & $21 \%$ & 1 & $\mathrm{~N}$ & $\mathrm{~N}$ & $Y t$ \\
\hline
\end{tabular}

Age: N/S, not summarised (tabulated data for every patient provided); question mark (?), not provided within the manuscript; N/A, not applicable. Statistical test result: $N$, not reported; $\mathrm{N}^{*}$, not reported but we identified the significant $p$ value from the original manuscript data. Severity of subject disease: The average clinical severity score of subjects with an index of spread listed in brackets. The number of severity groups which subjects were divided into is listed. ISS/AIS, Injury Severity Score/Abbreviated Injury Severity Score [87]; ASA, American Society of Anesthesiologists Physical Status Classification System [85]; APACHE II: Acute Physiology and Chronic Health Evaluation II [83], APACHE III, Acute Physiology and Chronic Health Evaluation III [84]. Subject drug use detailed: whether patient exposure to known immunomodulating drugs was documented. A ' $t$ ' signifies that the timing of the drug administration in relation to blood sampling was clear from the study methodology. 
Table 5 Demographic information of monocyte tolerance studies

\begin{tabular}{|c|c|c|c|c|c|c|c|c|c|c|c|c|c|}
\hline \multirow[t]{2}{*}{ Author } & \multicolumn{3}{|l|}{ Age } & \multicolumn{3}{|c|}{ Gender (\%male) } & \multirow{2}{*}{$\begin{array}{l}\text { Subject } \\
\text { ethnicity }\end{array}$} & \multicolumn{3}{|c|}{ Severity of subject disease } & \multicolumn{3}{|c|}{ Subject drug exposure documentation } \\
\hline & Subjects & Controls & $\begin{array}{l}\text { Statistical } \\
\text { test result }\end{array}$ & Subjects & Controls & $\begin{array}{l}\text { Statistical } \\
\text { test result }\end{array}$ & & Index & Score & $\begin{array}{l}\text { No. of } \\
\text { groups }\end{array}$ & Sedatives & Antibiotics & Steroids \\
\hline Liu [30] & $?$ & $?$ & $\mathrm{~N}$ & $?$ & $?$ & $\mathrm{~N}$ & $\mathrm{~N}$ & $?$ & $?$ & 1 & $\mathrm{~N}$ & $\mathrm{~N}$ & $\mathrm{~N}$ \\
\hline Buttenschoen [41] & 56 (33 to 88 ) & N/A & N & 70 & N/A & $\mathrm{N}$ & $\mathrm{N}$ & $?$ & $?$ & $\mathrm{n} / \mathrm{a}$ & $\mathrm{N}$ & N & $\mathrm{N}$ \\
\hline Pachot [31] & 68 (54 to 76$)$ & 51 (42 to 65$)$ & N & 62 & 52 & $\mathrm{~N}$ & $\mathrm{~N}$ & SAPS $\|$ & $51( \pm 5)$ & 2 & $\mathrm{~N}$ & $\mathrm{~N}$ & $\mathrm{~N}$ \\
\hline West [32] & $N / S$ & $N / S$ & N & 42 & $100 ; 20 ; 56$ & N & N & $?$ & $?$ & 2 & N & N & N \\
\hline Harter [33] & $48 \pm 20$ & 'Comparable' & $\mathrm{N}$ & 71 & 12 & $\mathrm{~N}$ & $\mathrm{~N}$ & APACHE ॥ & $13 \pm 6$ & 1 & $\mathrm{~N}^{\prime \prime}$ & $\mathrm{N}$ & $\mathrm{N}$ \\
\hline Flohe [40] & $47 \pm 18$ & $37 \pm 14$ & $\mathrm{~N}$ & 68 & 50 & $\mathrm{~N}$ & $\mathrm{~N}$ & ISS & $39 \pm 9$ & 1 & $\mathrm{~N}$ & $N$ & $\mathrm{~N}$ \\
\hline Escoll [34] & $51 \pm 12$ & $49 \pm 12$ & $\mathrm{~N}$ & $?$ & $?$ & $\mathrm{~N}$ & $\mathrm{~N}$ & $?$ & $?$ & 1 & $\mathrm{~N}$ & $\mathrm{~N}$ & $\mathrm{~N}$ \\
\hline Heagy [39] & $49 \pm 3 ; 44 \pm 8$ & $?$ & $N$ & $?$ & $?$ & $\mathrm{~N}$ & $\mathrm{~N}$ & Mortality & $20 \%, 9.6 \%$ & 2 & $\mathrm{~N}$ & $\mathrm{~N}$ & $\mathrm{~N}$ \\
\hline Calvano [35] & $60 ; 61$ & 58 & $\mathrm{~N}$ & $66 ; 66$ & 66 & N & $\mathrm{N}$ & $?$ & $?$ & 2 & $\mathrm{~N}$ & $\mathrm{~N}$ & $Y t$ \\
\hline Sfeir [36] & $63 \pm 3$ & $50 \pm 7$ & $N^{*}(p<0.0001)$ & 80 & 50 & $\mathrm{~N}$ & $\mathrm{~N}$ & APACHE ॥ & $27 \pm 5$ & 1 & $\mathrm{~N}$ & $\mathrm{~N}$ & $\mathrm{~N}$ \\
\hline Kawasaki [42] & $?$ & N/A & $\mathrm{N}$ & $?$ & N/A & $\mathrm{N}$ & $\mathrm{N}$ & ASA & | to || & 1 & $N$ & $\mathrm{~N}$ & $\mathrm{~N}$ \\
\hline Heagy [37] & $49 \pm 21$ & $?$ & N & 66 & $?$ & N & $\mathrm{N}$ & $?$ & $?$ & 4 & $N$ & $N$ & $\mathrm{~N}$ \\
\hline Bergmann [38] & $60 ; 51$ & 32 & $\mathrm{~N}$ & $?$ & $?$ & $\mathrm{~N}$ & $\mathrm{~N}$ & MODS & $15 \pm 1,7 \pm 1$ & 2 & $\mathrm{~N}$ & $\mathrm{~N}$ & $\mathrm{~N}$ \\
\hline
\end{tabular}

Age: N/S, not summarised (tabulated data for every patient provided); question mark (?), not provided within the manuscript; N/A, not applicable. Statistical test result: $\mathrm{N}$, not reported; $\mathrm{N}^{*}$, not reported but we

identified the significant $p$ value from the original manuscript data. Severity of subject disease: The average clinical severity score of subjects with an index of spread listed in brackets. The number of severity groups which subjects were divided into is listed. ISS/AIS, Injury Severity Score/Abbreviated Injury Severity Score [87]; ASA, American Society of Anesthesiologists Physical Status Classification System [85]; APACHE II: Acute Physiology and Chronic Health Evaluation II [83], APACHE III, Acute Physiology and Chronic Health Evaluation III [84]. Subject drug use detailed: whether patient exposure to known immunomodulating drugs was documented. A ' $t$ ' signifies that the timing of the drug administration in relation to blood sampling was clear from the study methodology. 
Table 6 Demographic information of lymphocyte apoptosis studies

\begin{tabular}{|c|c|c|c|c|c|c|c|c|c|c|c|c|c|}
\hline \multirow[t]{2}{*}{ Author } & \multicolumn{3}{|l|}{ Age } & \multicolumn{3}{|c|}{ Gender (\%male) } & \multirow[t]{2}{*}{ Ethnicity } & \multicolumn{3}{|c|}{ Severity of subject disease } & \multicolumn{3}{|c|}{$\begin{array}{l}\text { Subject drug exposure } \\
\text { documentation }\end{array}$} \\
\hline & Subjects & Controls & $\begin{array}{l}\text { Statistical } \\
\text { test result }\end{array}$ & Subjects & Controls & $\begin{array}{l}\text { Statistical } \\
\text { test result }\end{array}$ & & Index & Score & $\begin{array}{l}\text { No. of } \\
\text { groups }\end{array}$ & Sedatives & Antibiotics & Steroids \\
\hline Roger [43] & 63 (37 to 82$)$ & 55 (37 to 5) & 0.04 & 50 & 43 & 0.76 & $\mathrm{~N}$ & SAPS ॥ & 55 (12 to 92$)$ & 2 & N & Yt & $Y t$ \\
\hline $\begin{array}{l}\text { Bandyopadhyay } \\
\text { [58] }\end{array}$ & $?$ & 'Matched' & $\mathrm{N}$ & $?$ & 'Matched' & N & N & APACHE & $>21$ & 1 & N & N & N \\
\hline White [11] & 54 (72 to 80$)$ & $\begin{array}{l}\text { Bacteraemia: } \\
73 \text { (70 to } 82 \text { ) }\end{array}$ & $>0.05$ & 52 & $\begin{array}{l}\text { Bacteraemia } \\
40\end{array}$ & $>0.05$ & Y & APACHE & 25 (21 to 28$)$ & 2 & N & N & N \\
\hline White [11] & $64 \pm 2$ & $65 \pm 1$ & 0.74 & 68 & 70 & 0.86 & $\mathrm{~N}$ & & & 2 & N & N & N \\
\hline Zhang [44] & $58 \pm 4$ & $59 \pm 4$ & $\mathrm{~N}$ & 52 & 50 & $\mathrm{~N}$ & N & $\begin{array}{l}\text { APACHE } \\
\|\end{array}$ & $26 \pm 3$ & 1 & N & $Y t$ & $Y t$ \\
\hline Guignant [45] & 63 (54 to 73 ) & $?$ & $N$ & 68 & $N$ & $N$ & & SAPS ॥ & 53(39 to 64$)$ & 1 & N & N & $Y t$ \\
\hline Vaki [46] & $71 \pm 2$ & $?$ & $N$ & 54 & $?$ & $N$ & N & $\begin{array}{l}\text { APACHE } \\
\|\end{array}$ & $20 \pm 9$ & $1(3)$ & N & N & N \\
\hline Slotwinski [62] & $62 \pm 9 ; 63 \pm 9$ & - & $N$ & 5,50 & - & $N$ & N & TNM & $?$ & 1 & N & $Y t$ & N \\
\hline Gogos [47] & $\begin{array}{l}67 \pm 17 ; 68 \pm 20 \\
54 \pm 25 ; 64 \pm 16\end{array}$ & & $P<0.0001$ & $\begin{array}{l}52,62 \\
57,67,64\end{array}$ & & $P=0.011$ & N & $\begin{array}{l}\text { APACHE } \\
\|\end{array}$ & $\begin{array}{l}12 \pm 7 ; 16 \pm 9 ; \\
13 \pm 8 ; 18 \pm 8 ; 20 \pm 5\end{array}$ & 3 & N & N & N \\
\hline Hoogerwerf [48] & $57 \pm 5$ & $66 \pm 5$ & $\begin{array}{l}\mathrm{N}^{*} \\
(p<0.0001)\end{array}$ & 63 & 50 & $N$ & N & $\begin{array}{l}\text { APACHE } \\
\|\end{array}$ & $19 \pm 2$ & 1 & N & N & N \\
\hline Yousef [49] & $44 \pm 9$ & $45 \pm 9,44 \pm 10$ & N & 59 & 60,57 & $N$ & $N$ & SOFA & 12 (7 to 14$)$ & $3(5)$ & N & N & $N$ \\
\hline Turrel-Davin [50] & $60 \pm 4$ & 'Age matched' & $N$ & 63 & $\begin{array}{l}\text { 'Sex } \\
\text { matched' }\end{array}$ & $N$ & N & SAPS $\|$ & $51 \pm 3$ & 1 & N & N & Y \\
\hline Pelekanou [51] & $69 \pm 16$ & $64 \pm 20$ & 0.099 & 64 & 43 & 0.300 & $N$ & $\begin{array}{l}\text { APACHE } \\
\|\end{array}$ & $18 \pm 4 ; 15 \pm 5$ & 1 & $N$ & N & Y \\
\hline Papadima [61] & $66 \pm 7 ; 67 \pm 10$ & & 0.8 & 85,47 & & 0.54 & $N$ & ASA & | to || & 1 & $Y t$ & $Y t$ & $Y t$ \\
\hline Delogu [52] & $?$ & $?$ & $N$ & $?$ & $?$ & $N$ & N & $?$ & $?$ & 1 & N & N & N \\
\hline Weber [53] & $56 \pm 4$ & $61 \pm 5, ?$ & $>0.05$ & 68,80 & $?$ & $N$ & $N$ & SAPS ॥ & $26 \pm 2$ & 1 & $N$ & N & Y \\
\hline Roth [54] & $56 \pm 6$ & $52 \pm 14$ & $N$ & 66 & 'Matched' & $N$ & $N$ & APACHE & $N / S$ & 1 & $N$ & $N$ & $N$ \\
\hline
\end{tabular}


Table 6 Demographic information of lymphocyte apoptosis studies (Continued)

\begin{tabular}{|c|c|c|c|c|c|c|c|c|c|c|c|c|c|}
\hline Le Tulzo [55] & $55 \pm 4 ; 64 \pm 4$ & $72 \pm 4 ; 55 \pm 4$ & $\begin{array}{l}\mathrm{N}^{*} \\
(p<0.0001)\end{array}$ & $?$ & $?$ & $N$ & $\mathrm{~N}$ & SAPS $\|$ & $33 \pm 3 ; 58 \pm 4$ & 2 & $\mathrm{~N}$ & $\mathrm{~N}$ & $\mathrm{~N}$ \\
\hline Hotchkiss [56] & $N / S$ & $N / S$ & N & 59 & $56, ?$ & $N$ & N & - & & 1 & N & N & Y \\
\hline Delogu [63] & $47 \pm 17$ & 'Matched' & $\mathrm{N}$ & $?$ & 'Matched' & $N$ & $\mathrm{~N}$ & ASA & | to || & 1 & Y & $\mathrm{N}$ & $Y t$ \\
\hline Pellegrini [59] & $44(20-83)$ & (18 to 60 ) & N & $?$ & ? & $\mathrm{N}$ & N & ISS & 25 (9 to 59 ) & 1 & N & $\mathrm{N}$ & $\mathrm{N}$ \\
\hline Delogu [64] & $?$ & 'Matched' & N & $?$ & 'Matched' & N & N & ASA & | to || & 1 & N & N & $Y t$ \\
\hline Hotchkiss [60] & 18 to 46 & ? & N & 90 & ? & N & N & ISS & N/S (9 to 50$)$ & 1 & $\mathrm{~N}$ & N & $\mathrm{N}$ \\
\hline Hotchkiss [57] & $\mathrm{N} / \mathrm{S}$ & $N / S$ & N & 65 & $?$ & $\mathrm{~N}$ & N & - & & 1 & N & $\mathrm{N}$ & Y \\
\hline Sasajima [65] & $\begin{array}{l}62(55 \text { to } 74) ; \\
49(37 \text { to } 58)\end{array}$ & & N & & $?$ & $\mathrm{~N}$ & N & $?$ & $?$ & 1 & N & $N$ & $N$ \\
\hline Sugimoto [66] & $N / S$ & & N & 50 & & $\mathrm{~N}$ & $\mathrm{~N}$ & $?$ & $?$ & 1 & N & $\mathrm{N}$ & $\mathrm{Yt}$ \\
\hline
\end{tabular}

Age: N/S, not summarised (tabulated data for every patient provided); question mark (?), not provided within the manuscript; $\mathrm{N} / \mathrm{A}$, not applicable. Statistical test result: $\mathrm{N}$, not reported; $\mathrm{N}^{*}$, not reported but we

identified the significant $p$ value from the original manuscript data. Severity of subject disease: The average clinical severity score of subjects with an index of spread listed in brackets. The number of severity groups which subjects were divided into is listed. ISS/AIS, Injury Severity Score/Abbreviated Injury Severity Score [87]; ASA, American Society of Anesthesiologists Physical Status Classification System [85]; APACHE II: Acute Physiology and Chronic Health Evaluation II [83], APACHE III, Acute Physiology and Chronic Health Evaluation III [84]. Subject drug use detailed: whether patient exposure to known immunomodulating drugs was documented. A 't' signifies that the timing of the drug administration in relation to blood sampling was clear from the study methodology. 'Matched', paper provided no details but stated the control population was matched to the study population. 
Table 7 Experimental conduct and exclusion criteria of neutrophil respiratory burst studies

\begin{tabular}{|c|c|c|c|c|c|c|c|c|c|}
\hline \multirow[t]{2}{*}{ Author } & \multirow{2}{*}{$\begin{array}{l}\text { Study } \\
\text { population }\end{array}$} & \multicolumn{2}{|l|}{ Sample timing } & \multirow{2}{*}{$\begin{array}{l}\text { Definition } \\
\text { of sepsis }\end{array}$} & \multirow{2}{*}{$\begin{array}{l}\text { Microbiology } \\
\text { results } \\
\text { provided }\end{array}$} & \multirow{2}{*}{$\begin{array}{l}\text { Independent } \\
\text { adjudication } \\
\text { of sepsis d } \\
\text { iagnosis }\end{array}$} & \multirow{2}{*}{$\begin{array}{l}\text { Exclusion criteria } \\
\text { immunosuppressive } \\
\text { disease }\end{array}$} & \multirow{2}{*}{$\begin{array}{l}\text { Exclusion } \\
\text { criteria } \\
\text { malignancy }\end{array}$} & \multirow{2}{*}{$\begin{array}{l}\text { Primary conclusion of study } \\
\text { (in relation to neutrophil } \\
\text { respiratory burst) }\end{array}$} \\
\hline & & $\begin{array}{l}\text { Time of } \\
\text { first sample }\end{array}$ & $\begin{array}{l}\text { No. } \\
\text { samples } \\
\text { (time span) }\end{array}$ & & & & & & \\
\hline Santos [12] & Sepsis & $\begin{array}{l}72 \text { h (Dx sepsis); } \\
48 \text { h (organ failure); } \\
\text { onset of septic shock }\end{array}$ & 2 (7 days) & $1 \mathrm{~A}, \mathrm{~B}, \mathrm{C}$ & $N$ & $\mathrm{~N}$ & Y & Y & $\begin{array}{l}\text { Reactive oxygen species production by } \\
\text { neutrophils is increased in sepsis, and } \\
\text { [it] is associated with poor outcome }\end{array}$ \\
\hline $\begin{array}{l}\text { Gorgulu } \\
\text { [19] }\end{array}$ & Trauma & 24 h (Hosp Adm) & 1 & $2 A, B, C$ & $N$ & $N$ & Y & $N$ & $\begin{array}{l}\text { Fas stimulation of septic neutrophils } \\
\text { promotes apoptosis and inhibits functionality, } \\
\text { partially by non-apoptotic signalling }\end{array}$ \\
\hline Bruns [13] & $\begin{array}{l}\text { Sepsis } \\
\text { (cirrhotics) }\end{array}$ & $\begin{array}{l}24 \text { h } \\
\text { (Hosp Adm) }\end{array}$ & 1 & 5 & Y & $\mathrm{N}$ & Y & $\mathrm{N}$ & $\begin{array}{l}\text { [Within cirrhotic patients] augmented } \\
\text { neutrophil ROS release in response to } \\
\text { E. coli... becomes exhausted in the } \\
\text { presence of infection }\end{array}$ \\
\hline Shih [20] & Trauma & $\begin{array}{l}24 \mathrm{~h} \\
\text { (Hosp Adm) }\end{array}$ & 2 (3 days) & $\mathrm{N}$ & N & $\mathrm{N}$ & Y & Y & $\begin{array}{l}\text { Plasma migration inhibitory factor is one } \\
\text { of the important factors responsible } \\
\text { for early neutrophil activation }\end{array}$ \\
\hline Kasten [21] & Trauma & $\begin{array}{l}48 \text { to } 72 \mathrm{~h} \\
\text { (Post-trauma) }\end{array}$ & 1 & $N$ & N & $\mathrm{N}$ & Y & $\mathrm{N}$ & $\begin{array}{l}\text { Following trauma, there are concurrent } \\
\text { and divergent immunological responses... } \\
\text { hyper-inflammatory response by the } \\
\text { innate arm.... and hypo-inflammatory } \\
\text { response by the adaptive arm }\end{array}$ \\
\hline Valente [22] & Trauma & $\begin{array}{l}48 \mathrm{~h} \\
\text { (Hosp Adm) }\end{array}$ & 3 (5 days) & $\mathrm{N}$ & $N$ & $\mathrm{~N}$ & Y & $\mathrm{N}$ & $\begin{array}{l}\text { Injury results in differences in innate } \\
\text { immune function in the elderly when } \\
\text { compared with controls }\end{array}$ \\
\hline $\begin{array}{l}\text { Kawasaki } \\
{[26]}\end{array}$ & $\begin{array}{l}\text { Elective } \\
\text { surgery }\end{array}$ & Pre-insult & 5 (4 days) & $\mathrm{N}$ & $\mathrm{N}$ & $\mathrm{N}$ & Y & $\mathrm{N}$ & $\begin{array}{l}\text { The innate immune system is suppressed } \\
\text { from the early period of upper abdominal } \\
\text { surgery }\end{array}$ \\
\hline $\begin{array}{l}\text { Frohlich } \\
\text { [27] }\end{array}$ & $\begin{array}{l}\text { Elective } \\
\text { surgery }\end{array}$ & Pre-insult & $\begin{array}{l}2 \text { (end of } \\
\text { anaesth) }\end{array}$ & $N$ & $\mathrm{n} / \mathrm{a}$ & $\mathrm{n} / \mathrm{a}$ & Y & Y & $\begin{array}{l}\text { [This study demonstrates] suppression of } \\
\text { neutrophil function by propofol in vitro } \\
\text { [but not] in vivo }\end{array}$ \\
\hline Martins [14] & Sepsis & 48 h (ICU Adm) & 1 & $1 \mathrm{~B}, \mathrm{C}$ & Y & $\mathrm{N}$ & Y & Y & $\begin{array}{l}\text { Neutrophil function is enhanced } \\
\text { in patients with sepsis }\end{array}$ \\
\hline
\end{tabular}


Table 7 Experimental conduct and exclusion criteria of neutrophil respiratory burst studies (Continued)

\begin{tabular}{|c|c|c|c|c|c|c|c|c|c|}
\hline Barth [15] & Sepsis & $?$ & 6 (5 days) & $1 C(>4 d)$ & Y & $\mathrm{N}$ & $\mathrm{N}$ & $\mathrm{N}$ & $\begin{array}{l}\text { Endogenous G-CSF increases neutrophil } \\
\text { function in patients with severe sepsis } \\
\text { and septic shock }\end{array}$ \\
\hline Mariano [16] & $\begin{array}{l}\text { Sepsis (renal } \\
\text { replacement } \\
\text { therapy) }\end{array}$ & $?$ & 4 (1 day) & $1, B, D$ & $\mathrm{~N}$ & $\mathrm{~N}$ & $\mathrm{~N}$ & $\mathrm{~N}$ & $\begin{array}{l}\text { Sera from septic patients [demonstrate] an } \\
\text { enhanced priming activity on neutrophils } \\
\text { [that is] reduced by ultrafiltration }\end{array}$ \\
\hline Quaid [23] & Trauma & $\begin{array}{l}24 \text { h } \\
\text { (Hosp Adm) }\end{array}$ & 1 & N & $\mathrm{N}$ & $\mathrm{N}$ & $\mathrm{N}$ & $\mathrm{N}$ & $\begin{array}{l}\text { [After severe trauma] IL- } 8 \text { and GROa lose } \\
\text { the ability to regulate the TNFa induced } \\
\text { respiratory burst }\end{array}$ \\
\hline Wiezer [28] & $\begin{array}{l}\text { Elective } \\
\text { surgery }\end{array}$ & Pre-insult & 5 (7 days) & $\begin{array}{l}\text { "clinical } \\
\text { criteria" }\end{array}$ & $\mathrm{N}$ & N & Y & $\mathrm{N}$ & $\begin{array}{l}\text { Patients undergoing liver resection have } \\
\text { an increased activation of leukocytes } \\
\text { compared with other major abdominal } \\
\text { surgery [that is partially reversed] by } \\
\text { endotoxin neutralisation... with } \mathrm{rPPI}_{21}\end{array}$ \\
\hline Ahmed [17] & Sepsis & $\begin{array}{l}72 \mathrm{~h} \\
\text { (Proof of } \\
\text { infection) }\end{array}$ & 1 & $1 \mathrm{~A}, \mathrm{~B}$ & Y & Y & Y & Y & $\begin{array}{l}\text { Septic patients deliver fewer neutrophils to } \\
\text { secondary inflammatory sites }\end{array}$ \\
\hline Shih [29] & $\begin{array}{l}\text { Trauma/ } \\
\text { Surgery }\end{array}$ & 24 h (Hosp adm) & $3+(7$ days $)$ & $1 \mathrm{~A}, \mathrm{~B}, \mathrm{C}$ & $\mathrm{N}$ & N & Y & Y & $\begin{array}{l}\text { Surgery after [trauma] has no effect on the } \\
\text { priming of neutrophils }\end{array}$ \\
\hline Ertel [24] & Trauma & $\begin{array}{l}24 \mathrm{~h} \\
\text { (Hosp adm) }\end{array}$ & 2 (3 days) & $\mathrm{N}$ & $\mathrm{N}$ & N & Y & $\mathrm{N}$ & $\begin{array}{l}\text { Severe trauma stimulates acute-phase } \\
\text { priming in neutrophils }\end{array}$ \\
\hline Ogura [25] & Trauma & $\begin{array}{l}24 \mathrm{~h} \\
\text { (Post-trauma) }\end{array}$ & $4+1$ (21 days) & $2 \mathrm{ABC}$ & Y & N & $\mathrm{N}$ & $\mathrm{N}$ & $\begin{array}{l}\text { Severe trauma stimulates acute-phase } \\
\text { priming in neutrophils }\end{array}$ \\
\hline Pascual [18] & Sepsis & $\begin{array}{l}24 \mathrm{~h} \\
(\mathrm{ICU} \text { adm) }\end{array}$ & 1 & $1 \mathrm{AC}$ & Y & N & $\mathrm{N}$ & $\mathrm{N}$ & $\begin{array}{l}\text { Plasma of septic patients may have a } \\
\text { profound effect on neutrophil } \\
\text { response [and] differentiates } \\
\text { between sepsis and non-sepsis samples }\end{array}$ \\
\hline
\end{tabular}

Sample timing: Were control samples taken at the same time point after the inflammatory stimulus as subject samples? When was the first sample taken from the subject? How many samples were taken for each subject in total and over what time span? Sepsis criteria: The criteria used to enrol subjects into the study. Where subgroups of these criteria were used (e.g. septic shock) these are detailed. 0, not stated; 1, ACCP/SCCM 1992 Consensus Conference [73]; 2, ACCP/SCCM Consensus Conference 2001 [74]; 3, SSC Consensus Conference 2008 [75]; 4, CDC NNIC [86]; 5, Microbiology and clinical assessment; 6, Postmortem identification of infection; $\mathrm{N}$, infection not considered; question mark (?), criteria not described. Sepsis severity groups enrolled: $\mathrm{A}=$ sepsis, $\mathrm{B}=$ severe sepsis, $\mathrm{C}=$ septic shock, $\mathrm{D}=$ acute renal failure, $\mathrm{E}=\mathrm{SIRS}$. Microbiology documentation: Were causative organisms clearly isolated and identified? Were additional steps taken to define whether the subject had sepsis beyond the initial clinical diagnosis, i.e. retrospective review of the case in light of subsequent information? 
Table 8 Experimental conduct and exclusion criteria of monocyte tolerance studies

\begin{tabular}{|c|c|c|c|c|c|c|c|c|c|}
\hline \multirow[t]{2}{*}{ Author } & \multirow{2}{*}{$\begin{array}{l}\text { Study } \\
\text { population }\end{array}$} & \multicolumn{2}{|l|}{ Sample timing } & \multirow{2}{*}{$\begin{array}{l}\text { Definition } \\
\text { of sepsis }\end{array}$} & \multirow{2}{*}{$\begin{array}{l}\text { Microbiology } \\
\text { results } \\
\text { provided }\end{array}$} & \multirow{2}{*}{$\begin{array}{l}\text { Independent } \\
\text { adjudication } \\
\text { of sepsis } \\
\text { diagnosis }\end{array}$} & \multirow{2}{*}{$\begin{array}{l}\text { Exclusion criteria } \\
\text { immunosuppressive } \\
\text { disease }\end{array}$} & \multirow{2}{*}{$\begin{array}{l}\text { Exclusion } \\
\text { criteria } \\
\text { malignancy }\end{array}$} & \multirow{2}{*}{$\begin{array}{l}\text { Primary conclusion of study } \\
\text { (in relation to monocyte } \\
\text { endotoxin tolerance) }\end{array}$} \\
\hline & & $\begin{array}{l}\text { Time of } \\
\text { first } \\
\text { sample }\end{array}$ & $\begin{array}{l}\text { No. of } \\
\text { samples } \\
\text { (time span) }\end{array}$ & & & & & & \\
\hline Liu [30] & Sepsis & $?$ & 1 & ? BC & $\mathrm{N}$ & $\mathrm{N}$ & $\mathrm{N}$ & $\mathrm{N}$ & $\begin{array}{l}\text { TLR4 stimulation and human sepsis } \\
\text { activate pathways that couple NAD } \\
\text { and its sensor SIRT1 with epigenetic } \\
\text { reprogramming }\end{array}$ \\
\hline $\begin{array}{l}\text { Buttenschoen } \\
\text { [41] }\end{array}$ & Elective surgery & Pre-insult & 4 (2 days) & $\mathrm{N}$ & $\mathrm{N}$ & $\mathrm{N}$ & Y & $\mathrm{N}$ & $\begin{array}{l}\text { Cytokine liberation of mononuclear } \\
\text { cells suggests a state of postoperative } \\
\text { endotoxin tolerance }\end{array}$ \\
\hline Pachot [31] & Sepsis & $\begin{array}{l}72 \mathrm{~h} \\
\text { (onset sep shock) }\end{array}$ & 2 & $1 \mathrm{C}$ & Y & Y & $\mathrm{N}$ & $\mathrm{N}$ & $\begin{array}{l}\text { CX3CR1 expression [is] severely } \\
\text { down-regulated in [septic] monocytes } \\
\text { and associated with lack of functionality }\end{array}$ \\
\hline West [32] & Sepsis & $24 \mathrm{~h}(\mathrm{ICU} \mathrm{adm})$ & 1 & $1 \mathrm{~A}, \mathrm{E}$ & Y & N & $\mathrm{N}$ & $\mathrm{N}$ & $\begin{array}{l}\text { Leukocytes of septic patients, but not SIRS, } \\
\text { show LPS tolerance }\end{array}$ \\
\hline Harter [33] & Sepsis & $?$ & 1 & $1 A B C$ & Y & Y & $\mathrm{N}$ & $\mathrm{N}$ & $\begin{array}{l}\text { Endotoxin tolerance in septic patients } \\
\text { does not depend solely on TLR-2 or } \\
\text { TLR- } 4 \text { expression }\end{array}$ \\
\hline Flohe [40] & $\begin{array}{l}\text { Surgery in } \\
\text { trauma patients }\end{array}$ & $48 \mathrm{~h}$ (ICU adm) & Mon, Thu. & $1 A B C$ & Y & $\mathrm{N}$ & Y & Y & $\begin{array}{l}\text { Initial trauma [and] major secondary surgery } \\
\text { cause suppression of immune functions, } \\
\text { whereas minor surgery does not }\end{array}$ \\
\hline Escoll [34] & Sepsis & $\begin{array}{l}48 \mathrm{~h} \\
\text { (onset sepsis) }\end{array}$ & 1 & $1 \mathrm{~A}$ & Y & Y & Y & Y & $\begin{array}{l}\text { Monocytes from septic patients rapidly express } \\
\text { IRAK-M mRNA when stimulated with LPS } \\
\text { ex vivo [unlike healthy volunteers] }\end{array}$ \\
\hline Heagy [39] & $\begin{array}{l}\text { ICU patients } \\
\text { (sepsis) }\end{array}$ & $72 \mathrm{~h}(\mathrm{ICU}$ adm) & 1 & 5 & $\mathrm{~N}$ & Y & $\mathrm{N}$ & $\mathrm{N}$ & $\begin{array}{l}\text { ICU patients with...endotoxin tolerance have } \\
\text { significantly poorer clinical outcomes }\end{array}$ \\
\hline Calvano [35] & Sepsis & $?$ & 1 & $1 \mathrm{EA}$ & Y & N & $N$ & N & $\begin{array}{l}\text { Cellular LPS hyporesponsiveness [cannot] be } \\
\text { ascribed to significant alterations in... } \\
\text { cell surface LPS binding proteins }\end{array}$ \\
\hline
\end{tabular}


Table 8 Experimental conduct and exclusion criteria of monocyte tolerance studies (Continued)

\begin{tabular}{lllll}
\hline Sfeir [36] & Sepsis & 24 (Sep Shock) & 1 & $1 \mathrm{C}$
\end{tabular}


Table 9 Experimental conduct and exclusion criteria of lymphocyte apoptosis studies

\begin{tabular}{|c|c|c|c|c|c|c|c|c|c|}
\hline \multirow[t]{2}{*}{ Author } & \multirow{2}{*}{$\begin{array}{l}\text { Study } \\
\text { population }\end{array}$} & \multicolumn{2}{|l|}{ Sample timing } & \multirow{2}{*}{$\begin{array}{l}\text { Definition } \\
\text { of sepsis }\end{array}$} & \multirow{2}{*}{$\begin{array}{l}\text { Microbiology } \\
\text { results } \\
\text { provided }\end{array}$} & \multirow{2}{*}{$\begin{array}{l}\text { Independent } \\
\text { adjudication } \\
\text { of sepsis } \\
\text { diagnosis }\end{array}$} & \multirow{2}{*}{$\begin{array}{l}\text { Exclusion criteria } \\
\text { immunosuppressive } \\
\text { disease }\end{array}$} & \multirow{2}{*}{$\begin{array}{l}\text { Exclusion } \\
\text { criteria } \\
\text { malignancy }\end{array}$} & \multirow{2}{*}{$\begin{array}{l}\text { Primary conclusion of study } \\
\text { (in relation to lymphocyte } \\
\text { apoptosis) }\end{array}$} \\
\hline & & $\begin{array}{l}\text { Time of } \\
\text { first sample }\end{array}$ & $\begin{array}{l}\text { No. samples } \\
\text { (time span) }\end{array}$ & & & & & & \\
\hline Roger [43] & Sepsis & Before first abs & 1 & $3 B C$ & Y & Y & Y & Y & $\begin{array}{l}\text { Concomitant } \mathrm{T} \text { cell proliferation and } \mathrm{T} \text { cell } \\
\text { apoptosis are observed in human sepsis }\end{array}$ \\
\hline $\begin{array}{l}\text { Bandyopadhyay } \\
\text { [58] }\end{array}$ & Trauma & $?$ & $\begin{array}{l}\text { Every } 4 \text { days } \\
\text { ( } 28 \text { days) }\end{array}$ & N & $N$ & $\mathrm{~N}$ & Y & N & $\begin{array}{l}\text { CD47 triggering, SHP-1 mediated NFkB } \\
\text { suppression and elevated TRAIL levels } \\
\text { increase... T cell apoptosis }\end{array}$ \\
\hline White [11] & Sepsis & $\begin{array}{l}24 \mathrm{~h} \text { (ICU } \\
\text { adm/positive BC) }\end{array}$ & 2 (7 days) & $1 \mathrm{BC}$ & $N$ & Y & Y & $\mathrm{N}$ & $\begin{array}{l}\text { Patients with infection and sepsis have } \\
\text { deficient IL-2 and IL-7 gene expression }\end{array}$ \\
\hline White [11] & $\begin{array}{l}\text { Elective } \\
\text { surgery } \\
\text { (infective } \\
\text { complications) }\end{array}$ & Pre-insult & 3 (5 days) & 4 & N & Y & Y & $N$ & \\
\hline Zhang [44] & Sepsis & 24 h (sep shock) & 1 & $1 C$ & N & N & Y & N & $\begin{array}{l}\text { The expression of PD-1 on T cells [is] up } \\
\text { regulated in septic shock }\end{array}$ \\
\hline Guignant [45] & Sepsis & 48 h (sep shock) & 3 (10 days) & $1 \mathrm{C}$ & Y & Y & $\mathrm{N}$ & Y & $\begin{array}{l}\text { PD-1 related molecules may constitute } \\
\text { a novel immunoregulatory system involved in } \\
\text { sepsis-induced immune alterations }\end{array}$ \\
\hline Vaki [46] & Sepsis & $\begin{array}{l}12 \mathrm{~h} \\
\text { (organ failure) }\end{array}$ & & $2 B C$ & Y & Y & Y & $\mathrm{N}$ & $\begin{array}{l}\text { These findings support...the existence of an } \\
\text { early circulating factor in severe sepsis/shock, } \\
\text { modulating apoptosis of CD4 lymphocytes }\end{array}$ \\
\hline Slotwinski [62] & $\begin{array}{l}\text { Elective } \\
\text { surgery }\end{array}$ & Pre-insult & 4 (7 days) & $\mathrm{N}$ & N & N & Y & $N$ & $\begin{array}{l}\text { Preoperative enteral immunonutrition prevents } \\
\text { postoperative decrease in lymphocyte subsets }\end{array}$ \\
\hline Gogos [47] & Sepsis & $\begin{array}{l}24 \mathrm{~h} \\
\text { (signs of sepsis) }\end{array}$ & 1 & $2 B C$ & Y & Y & Y & $N$ & $\begin{array}{l}\text { Major differences of the early statuses of innate } \\
\text { and adaptive immune systems exist between } \\
\text { sepsis and severe sepsis/shock in relation the } \\
\text { underlying type of infection }\end{array}$ \\
\hline Hoogerwerf [48] & Sepsis & $\begin{array}{l}24 \mathrm{~h} \\
\text { (dx sepsis) }\end{array}$ & 1 & $2 \mathrm{~A}$ & Y & Y & Y & $\mathrm{N}$ & $\begin{array}{l}\text { In patients with sepsis, alterations in apoptosis } \\
\text { of circulating leukocytes occur in a cell-specific } \\
\text { manner }\end{array}$ \\
\hline
\end{tabular}


Table 9 Experimental conduct and exclusion criteria of lymphocyte apoptosis studies (Continued)

\begin{tabular}{|c|c|c|c|c|c|c|c|c|c|}
\hline Yousef [49] & Sepsis & $?$ & 1 & $1 \mathrm{ABC}$ & N & N & Y & $\mathrm{N}$ & $\begin{array}{l}\text { Percentage of apoptotic lymphocyte median } \\
\text { values [could be] an indicator of prognosis } \\
\text { and survival in critically ill patients }\end{array}$ \\
\hline Turrel-Davin [50] & Sepsis & $\begin{array}{l}48 \mathrm{~h} \\
\text { (sep shock) }\end{array}$ & 2 (5 days) & $1 \mathrm{C}$ & Y & Y & $\mathrm{N}$ & $\mathrm{N}$ & $\begin{array}{l}\text { Pro-apoptotic genes BID and FAS appear to } \\
\text { constitute promising apoptosis markers }\end{array}$ \\
\hline Pelekanou [51] & Sepsis & $\begin{array}{l}24 \mathrm{~h} \\
\text { (signs of sepsis) }\end{array}$ & 1 & $12 \mathrm{ABC}$ & Y & Y & Y & N & $\begin{array}{l}\text { Decrease of CD-4 lymphocytes... } \\
\text { is characteristic of sepsis arising in } \\
\text { ventilator associated pneumonia }\end{array}$ \\
\hline Papadima [61] & $\begin{array}{l}\text { Elective } \\
\text { surgery }\end{array}$ & Pre-insult & 2 (1 day) & $\mathrm{N}$ & - & & Y & Y & $\begin{array}{l}\text { No alterations in lymphocyte counts [and] } \\
\text { subpopulations [following use of epidural } \\
\text { anaesthesia] }\end{array}$ \\
\hline Delogu [52] & Sepsis & $\begin{array}{l}24 \mathrm{~h} \\
\text { (sep shock) }\end{array}$ & 1 & $? \mathrm{C}$ & Y & N & $\mathrm{N}$ & $\mathrm{N}$ & $\begin{array}{l}\text { Blood caspase-1 elevated in sepsis. IL-6 } \\
\text { correlates with apoptotic rate and caspase-9 } \\
\text { expression in lymphocytes }\end{array}$ \\
\hline Weber [53] & Sepsis & $4 \mathrm{~h}$ (sev sepsis) & 1 & $1 \mathrm{~B}$ & $\mathrm{~N}$ & N & Y & Y & $\begin{array}{l}\text { In early severe sepsis....induction of... } \\
\text { Bim,Bid,Bak and downregulation of } \mathrm{BCl}-2 \\
\text { and } \mathrm{BCl}-\mathrm{xl} \text { is observed }\end{array}$ \\
\hline Roth [54] & Sepsis & $?$ & 1 & $1 \mathrm{ABC}$ & $\mathrm{N}$ & N & $\mathrm{N}$ & $\mathrm{N}$ & $\begin{array}{l}\text { These findings strongly suggest that in septic } \\
\text { patients Th1 T cells are selectively susceptible } \\
\text { to apoptosis }\end{array}$ \\
\hline Le Tulzo [55] & Sepsis & $\begin{array}{l}+ \text { ve } \\
\text { microbiology } \\
\pm 3 \text { days }\end{array}$ & 2 (6 days) & $1 \mathrm{BCE}$ & Y & N & $\mathrm{N}$ & $\mathrm{N}$ & $\begin{array}{l}\text { Lymphocyte apoptosis is rapidly increased in... } \\
\text { septic shock...and leads to a profound and } \\
\text { persistent lymphopaenia associated with } \\
\text { poor outcome }\end{array}$ \\
\hline Hotchkiss [56] & Sepsis & 6 h (death) & 1 & 6 & Y & $\mathrm{N}$ & Y & $\mathrm{N}$ & $\begin{array}{l}\text { Capsase } 9 \text { mediates profound progressive loss } \\
\text { of B and CD4 T helper cells in [severe] sepsis }\end{array}$ \\
\hline Delogu [63] & $\begin{array}{l}\text { Elective } \\
\text { surgery }\end{array}$ & Pre-insult & 3 (4 days) & $\mathrm{N}$ & $N$ & $\mathrm{~N}$ & Y & Y & $\begin{array}{l}\text { Surgical trauma is associated with a significant } \\
\text { but transient increase in lymphocyte } \\
\text { commitment to apoptosis }\end{array}$ \\
\hline Pellegrini [59] & Trauma & $?$ & $\begin{array}{l}\text { 2/week } \\
\text { (until death/ } \\
\text { discharge) }\end{array}$ & $\mathrm{N}$ & N & N & $\mathrm{N}$ & N & $\begin{array}{l}\text { Increased levels of apoptosis are not directly } \\
\text { associated with negative trauma patient } \\
\text { outcome }\end{array}$ \\
\hline
\end{tabular}


Table 9 Experimental conduct and exclusion criteria of lymphocyte apoptosis studies (Continued)

\begin{tabular}{|c|c|c|c|c|c|c|c|c|c|}
\hline Delogu [64] & Surgical & Pre-insult & 3 (4 days) & $\mathrm{N}$ & N & N & Y & Y & $\begin{array}{l}\text { Surgical trauma upregulates lymphocyte death } \\
\text { signalling factors and downregulates survival } \\
\text { factors. Increased apoptosis of CD8+ cells } \\
\text { maybe associated with greater risk of } \\
\text { postsurgical infection }\end{array}$ \\
\hline Hotchkiss [60] & Trauma & $\begin{array}{l}10 \mathrm{~h} \\
\text { (injury to surgery) }\end{array}$ & 1 & $\mathrm{~N}$ & N & N & $\mathrm{N}$ & N & $\begin{array}{l}\text { Focal apoptosis of intestinal epithelial and } \\
\text { lymphoid tissues occurs extremely rapidly after } \\
\text { injury }\end{array}$ \\
\hline Hotchkiss [57] & Sepsis & 6 h (death) & 1 & 6 & Y & Y & N & N & $\begin{array}{l}\text { Caspase- } 3 \text { mediated apoptosis causes } \\
\text { extensive lymphocyte apoptosis in sepsis }\end{array}$ \\
\hline Sasajima [65] & $\begin{array}{l}\text { Elective } \\
\text { surgery }\end{array}$ & Pre-insult & 5 (7 days) & $\mathrm{N}$ & N & N & N & N & $\begin{array}{l}\text { Transient T cell apoptosis occurs after major } \\
\text { operations }\end{array}$ \\
\hline Sugimoto [66] & $\begin{array}{l}\text { Elective } \\
\text { surgery }\end{array}$ & Pre-insult & 4 (4 days) & $\mathrm{N}$ & N & N & N & N & $\begin{array}{l}\text { Enhanced FasL expression is likely to be } \\
\text { related to systemic inflammatory responses } \\
\text { induced during the perioperative period }\end{array}$ \\
\hline
\end{tabular}




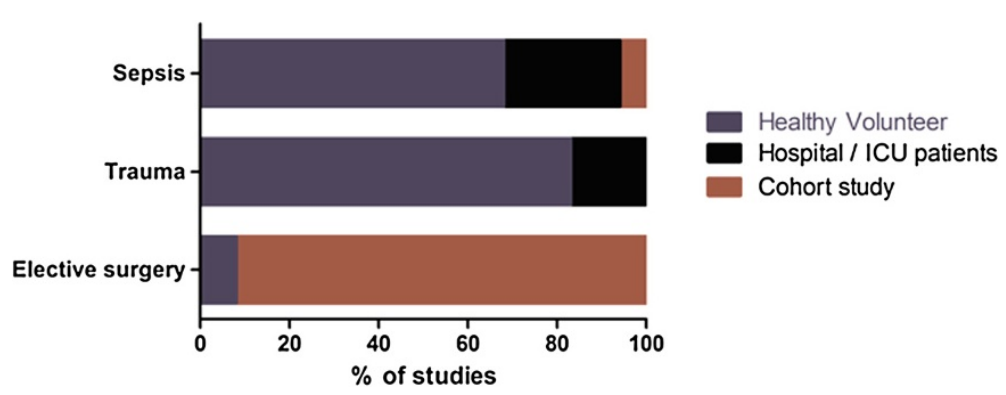

Figure 2 Identification of experimental control groups. The specific details for Hospital/ICU patients are detailed within Tables 1,2 and 3. Within cohort study pre-insult baseline samples were taken from the study population, allowing them to act as their own experimental control.

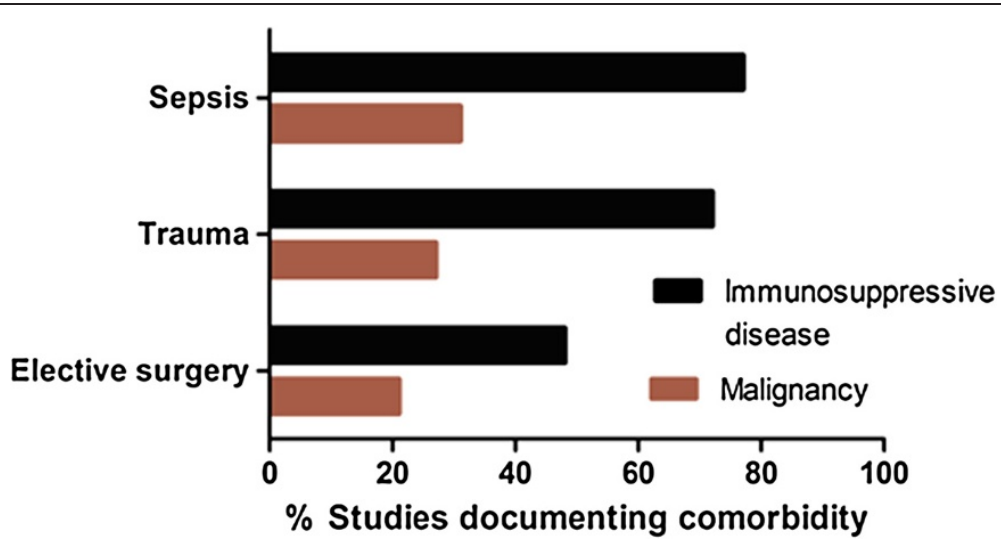

Figure 3 Documentation of patients' comorbid disease.

comparable between studies and was most frequently defined by the severity of sepsis (Figure 4). These triggers included hospital admission (1/26), ICU admission (5/26), proof of infection $(2 / 26)$, diagnosis of sepsis $(5 / 26)$, onset of sepsis $(14 / 26 ; 54 \%)$, onset of organ failure (3/24) and onset of septic shock (7/26) - the remaining two samples were from autopsy studies. Multiple criteria for sampling were often used and

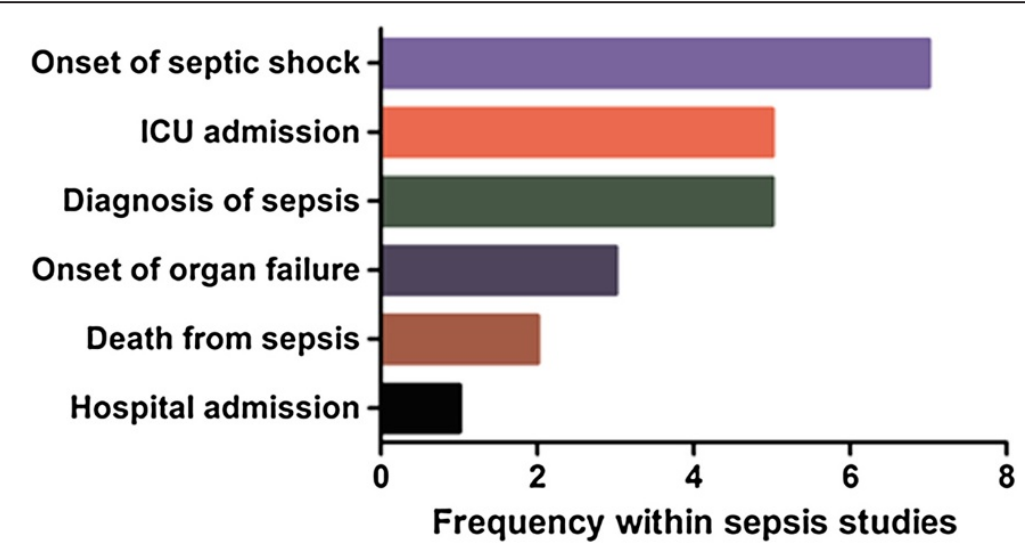

Figure 4 Event trigger used for index blood sample to be taken within studies of septic patients. 
dependent upon the severity of patient illness. Approximately half of all studies (14/26; $58 \%$ ) obtained an initial sample within $24 \mathrm{~h}$ of hospital admission. Similar patterns of sample timing were described for trauma patients. Repeat samples were often undertaken, but over highly variable intervals that were frequently not defined a priori. By contrast, all 12 studies undertaken in the elective surgical setting obtained preoperative control samples, with subsequent samples taken on predefined postoperative days.

\section{Therapies as potential confounders}

Commonly administered therapies in intensive care impact directly on immune function [8-10]. We assessed reporting of three of the commonest therapies with established immunomodulatory properties and found that only up to a quarter of studies documented their use (Figure 5). Specifically, these were sedative agents $(4 / 57 ; 7 \%)$, antibiotics $(6 / 57 ; 11 \%)$ and steroids $(15 / 57 ; 26 \%)$.

\section{Experimental conduct and outcomes}

There was no apparent relationship between the experimental context of studies and the control groups that were explored (Tables 1, 2 and 3). There are, however, clear associations between the study population studied and experimental outcome (Tables 1, 7, 2, 8, 3 and 9). For example, within the respiratory burst data, there is a consistent increase in respiratory burst identified by sepsis studies. However, since none of these studies used pre-illness samples, it is unclear if the change is a feature of sepsis, or the study population in relation to healthy volunteers. The conflicting results reported by the three surgical studies are difficult to interpret since each study uses a different burst assay, and the magnitude/type of operation varies. Similar patterns are also evident across the monocyte and lymphocyte studies.

\section{Discussion}

This systematic review has revealed several important issues in the design and reporting of immunologic phenotype in intensive care/sepsis studies. The studies we selected are representative of the current literature, covering the past 15 years of work in three key areas of sepsis research. Following a preliminary Pubmed search,

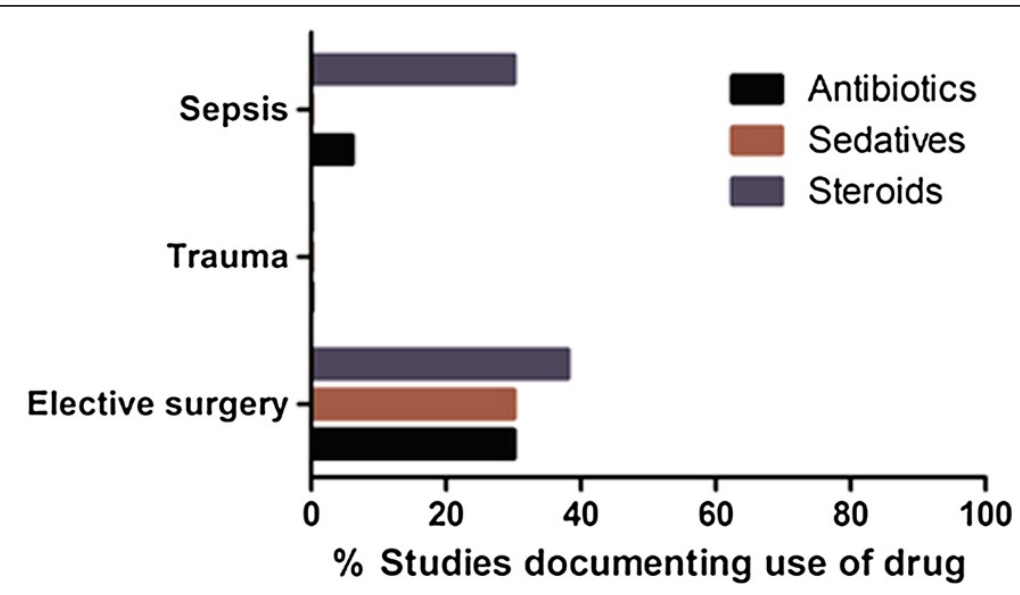

Figure 5 Documentation of drug exposure of the study population. 
these three assays were chosen because they represent the most frequently investigation for each immune cell type. These limitations refer to the clinical aspects of the study methodology rather than specific laboratory techniques that we did not assess. These data suggest that the use of surgical patients to model critical illness may overcome several key limitations.

Defining what constitutes an adequate control sample for the immunologic study of sepsis is clearly highly challenging. Case-control studies are frequently used in sepsis research. Our review suggests that case-control studies cannot easily determine whether the observed differences in the experimental readout between the study and control groups is due to sepsis per se, or other differences between the groups including age, comorbidities and treatment interventions. Whereas cohort studies do allow pre-sepsis samples to be taken, the majority of studies are conducted in healthy volunteers free of important comorbidities (e.g. heart failure, cirrhosis) that influence both the development of, and survival from, sepsis [71]. Furthermore, age-, gender- and ethnicity-related differences in immune function are well documented [67-70], yet our data demonstrates that several key demographic details for study and control populations were frequently not reported. Finally, the presence of malignant disease - associated with immunosuppression [72] and disproportionately represented in the ICU population of most healthcare systems - was only documented in a minority of studies.

Sepsis is currently defined using clinical constructs that define syndromes, rather than use biologic and/or molecular criteria. It remains unclear whether there are biologically relevant differences between clinically defined subtypes of sepsis. In other words, changes in immunophenotype associated with progression of sepsis to severe sepsis/septic shock may merely reflect the consequences of clinical interventions and/or indirect effects on organ function that partly reflect pre-existing comorbidities. Furthermore, the specific detection of pathogens, or pathogen-associated molecular patterns, is likely to further impact on the robustness of immunophenotyping since the location and type of micro-organism both regulate host-immune responses $[77,78]$. We identified only one study that specified infection site and/or a specific pathogen [34].

Critically ill patients are exposed to a range of therapeutic agents that have well-described immunologic effects. Although immunomodulation by the majority of these agents has been established in vitro, their role in confounding the septic immunophenotype remains unclear. Nevertheless, a myriad of off-target, immune effects have been established in pre-clinical in vivo models. Many antibiotics target mitochondria and eukaryotic protein synthesis [79]. Steroids exert potent pro- and anti-inflammatory properties - including inducing lymphocyte apoptosis [9]. Similarly, sedatives and analgesics exert profound effects on immune cell function [80,81].

Our data suggest that surgical patients offer important potential advantages for mechanistic studies of sepsis. The incidence of sepsis - as defined by conventional clinical criteria - varies from $6.98 \%$ to $12.25 \%$, depending upon the health care system and database interrogated [82]. No other patient population allows the collection of highly phenotyped data and individualised control samples prior to a defined traumatic insult. Since the volume of surgery is huge and large scale outcome data can be collected, potential limitations including comorbidities and concomitant therapies can be controlled for. 


\section{Conclusions}

We found several important limitations in clinical design associated with translational immunologic studies of human sepsis. Clinical design in mechanistic studies exploring changes in immunophenotype may contribute to the lack of translational therapeutic progress in intensive care medicine. Major elective surgery offers a potential model to overcome many of these methodological limitations.

\section{Take-home message}

Systematic review suggests a critical re-evaluation in design of immunologic phenotyping studies conducted in intensive care.

\section{Tweet}

Immunological investigation of septic patients presents methodological challenges that are not considered by many recent studies.

\section{Abbreviations}

ACCP/SCCM: American College of Chest Physicians/Society of Critical Care Medicine; APACHE II: Acute Physiology and Chronic Health Evaluation [83]; APACHE III: Acute Physiology and Chronic Health Evaluation [84]; ASA: American Society of Anesthesiologists [85]; BC: Blood culture; CDC NNIS: Centre for Disease Control National Nosocomial Infections Surveillance [86]; ICU: Intensive Care Unit; ISS: Injury Severity Score [87]; MODS: Multi Organ Dysfunction Score; N/A: not applicable; N/S: not summarised; SAPS II: Simplified Acute Physiology Score; SSC: Surviving Sepsis Campaign; SIRS: Systemic Inflammatory Response Syndrome.

\section{Competing interests}

GLA is supported by an Academy of Medical Science/Health Foundation Clinician Scientist Award. This work was undertaken at UCLH/UCL which received a proportion of funding from the Department of Health's NIHR Biomedical Research Centres funding scheme.

\section{Authors' contributions}

DC performed literature search, compiled data tables plus results and drafted the manuscript. AGDA designed the study and drafted/revised manuscript. GLA designed the study and drafted/revised manuscript. All authors read and approved the final manuscript.

Received: 4 November 2013 Accepted: 21 January 2014

Published: 27 February 2014

\section{References}

1. Harrison DA, Welch CA, Eddleston JM (2006) The epidemiology of severe sepsis in England, Wales and Northern Ireland, 1996 to 2004: secondary analysis of a high quality clinical database, the ICNARC Case Mix Programme Database. Crit Care 10:R42

2. Dombrovskiy VY, Martin AA, Sunderram J, Paz HL (2007) Rapid increase in hospitalization and mortality rates for severe sepsis in the United States: a trend analysis from 1993 to 2003. Crit Care Med 35:1244-1250

3. Ranieri VM, Thompson BT, Barie PS, Dhainaut J-F, Douglas IS, Finfer S, Gårdlund B, Marshall JC, Rhodes A, Artigas A, Payen D, Tenhunen J, Al-Khalidi HR, Thompson V, Janes J, Macias WL, Vangerow B, Williams MD (2012) Drotrecogin Alfa (activated) in adults with septic shock. N Engl J Med 366:2055-2064

4. Sprung CL, Annane D, Keh D, Moreno R, Singer M, Freivogel K, Weiss YG, Benbenishty J, Kalenka A, Forst H, Laterre P-F, Reinhart K, Cuthbertson BH, Payen D, Briegel J (2008) Hydrocortisone therapy for patients with septic shock. N Engl J Med 358:111-124

5. Finfer S, Chittock DR, Su SY-S, Blair D, Foster D, Dhingra V, Bellomo R, Cook D, Dodek P, Henderson WR, Hébert PC, Heritier S, Heyland DK, McArthur C, McDonald E, Mitchell I, Myburgh JA, Norton R, Potter J, Robinson BG, Ronco JJ (2009) Intensive versus conventional glucose control in critically ill patients. N Engl J Med 360:1283-1297

6. Mestas J, Hughes CCW (2004) Of mice and not men: differences between mouse and human immunology. J Immunol 172:2731-2738

7. Xiao W, Mindrinos MN, Seok J, Cuschieri J, Cuenca AG, Gao H, Hayden DL, Hennessy L, Moore EE, Minei JP, Bankey PE, Johnson JL, Sperry J, Nathens AB, Billiar TR, West MA, Brownstein BH, Mason PH, Baker HV, Finnerty CC, Jeschke MG, López MC, Klein MB, Gamelli RL, Gibran NS, Arnoldo B, Xu W, Zhang Y, Calvano SE, McDonald-Smith GP et al (2011) A genomic storm in critically injured humans. J Exp Med 208:2581-2590

8. Kanoh S, Rubin BK (2010) Mechanisms of action and clinical application of macrolides as immunomodulatory medications. Clin Microbiol Rev 23:590-615

9. Clarke AR, Purdie CA, Harrison DJ, Morris RG, Bird CC, Hooper ML, Wyllie AH (1993) Thymocyte apoptosis induced by p53-dependent and independent pathways. Nature 362:849-852

10. Mikawa K, Akamatsu H, Maekawa N, Nishina K, Niwa Y (1998) Propofol inhibits human neutrophil functions. Anesth Analg 87:695-700 
11. White M, Mahon V, Grealy R, Doherty DG, Stordeur P, Kelleher DP, McManus R, Ryan T (2011) Post-operative infection and sepsis in humans is associated with deficient gene expression of $ү c$ cytokines and their apoptosis mediators. Crit Care 15:R158

12. Santos SS, Brunialti MKC, Rigato O, Machado FR, Silva E, Salomao R (2012) Generation of nitric oxide and reactive oxygen species by neutrophils and monocytes from septic patients and association with outcomes. Shock 38:18-23

13. Bruns T, Peter J, Hagel S, Herrmann A, Stallmach A (2011) The augmented neutrophil respiratory burst in response to Escherichia coli is reduced in liver cirrhosis during infection. Clin Exp Immunol 164:346-356

14. Martins PS, Kallas EG, Neto MC, Dalboni MA, Blecher S, Salomão R (2003) Upregulation of reactive oxygen species generation and phagocytosis, and increased apoptosis in human neutrophils during severe sepsis and septic shock. Shock 20:208-212

15. Barth E, Fischer G, Schneider EM, Moldawer LL, Georgieff M, Weiss M (2002) Peaks of endogenous G-CSF serum concentrations are followed by an increase in respiratory burst activity of granulocytes in patients with septic shock. Cytokine 17:275-284

16. Mariano F, Tetta C, Guida G, Triolo G, Camussi G (2001) Hemofiltration reduces the serum priming activity on neutrophil chemiluminescence in septic patients. Kidney Int 60:1598-1605

17. Ahmed NA, McGill S, Yee J, Hu F, Michel RP, Christou NV (1999) Mechanisms for the diminished neutrophil exudation to secondary inflammatory sites in infected patients with a systemic inflammatory response (sepsis). Crit Care Med 27:2459-2468

18. Pascual C, Bredle D, Karzai W, Meier-Hellmann A, Oberhoffer M, Reinhart K (1998) Effect of plasma and LPS on respiratory burst of neutrophils in septic patients. Intensive Care Med 24:1181-1186

19. Paunel-Görgülü A, Lögters T, Flohé S, Cinatl J, Altrichter J, Windolf J, Scholz M (2011) Stimulation of Fas signaling down-regulates activity of neutrophils from major trauma patients with SIRS. Immunobiology 216:334-342

20. Shih H-C, Huang M-S, Lee C-H (2010) Polymorphonuclear cell priming associated with NF-kB activation in patients with severe injury is partially dependent on macrophage migration inhibitory factor. J Am Coll Surg 211:791-797

21. Kasten KR, Goetzman HS, Reid MR, Rasper AM, Adediran SG, Robinson CT, Cave CM, Solomkin JS, Lentsch AB, Johannigman JA, Caldwell CC (2010) Divergent adaptive and innate immunological responses are observed in humans following blunt trauma. BMC Immunol 11:4

22. Valente SA, Fallon WF, Alexander TS, Tomas ER, Evancho-Chapman MM, Schmidt SP, Gorski R, Pizov O, DeFine L, Clark AJ (2009) Immunologic function in the elderly after injury-the neutrophil and innate immunity. J Trauma 67:968-974

23. Quaid G, Cave C, Williams MA, Hennigan RF, Bokoch G, Solomkin JS (2001) Mechanisms of human neutrophil oxidant production after severe injury. Surgery 130:669-675, discussion 675-6

24. Ertel W, Keel M, Buergi U, Hartung T, Imhof HG, Trentz O (1999) Granulocyte colony-stimulating factor inhibits neutrophil apoptosis at the local site after severe head and thoracic injury. J Trauma 46:784-792, discussion 792-3

25. Ogura H, Tanaka H, Koh T, Hashiguchi N, Kuwagata Y, Hosotsubo H, Shimazu T, Sugimoto H (1999) Priming, second-hit priming, and apoptosis in leukocytes from trauma patients. J Trauma 46:774-781, discussion 781-3

26. Kawasaki T, Ogata M, Kawasaki C, Okamoto K, Sata T (2007) Effects of epidural anaesthesia on surgical stress-induced immunosuppression during upper abdominal surgery. Br J Anaesth 98:196-203

27. Fröhlich D, Trabold B, Rothe G, Hoerauf K, Wittmann S (2006) Inhibition of the neutrophil oxidative response by propofol: preserved in vivo function despite in vitro inhibition. Eur J Anaesthesiol 23:948-953

28. Wiezer MJ, Meijer C, Sietses C, Prins HA, Cuesta MA, Beelen RH, Meijer S, van Leeuwen PA (2000) Bactericidal/ permeability-increasing protein preserves leukocyte functions after major liver resection. Ann Surg 232:208-215

29. Shih HC, Su CH, Lee CH (1999) Superoxide production of neutrophils after severe injury: impact of subsequent surgery and sepsis. Am J Emerg Med 17:15-18

30. Liu TF, Yoza BK, El Gazzar M, Vachharajani VT, McCall CE (2011) NAD + -dependent SIRT1 deacetylase participates in epigenetic reprogramming during endotoxin tolerance. J Biol Chem 286:9856-9864

31. Pachot A, Cazalis M-A, Venet F, Turrel F, Faudot C, Voirin N, Diasparra J, Bourgoin N, Poitevin F, Mougin B, Lepape A, Monneret G (2008) Decreased expression of the fractalkine receptor CX3CR1 on circulating monocytes as new feature of sepsis-induced immunosuppression. J Immunol 180:6421-6429

32. West MA, Koons A, Crandall M, Skinner R, Worley M, Shapiro MB (2007) Whole blood leukocyte mitogen activated protein kinases activation differentiates intensive care unit patients with systemic inflammatory response syndrome and sepsis. J Trauma 62:805-811

33. Härter L, Mica L, Stocker R, Trentz O, Keel M (2004) Increased expression of toll-like receptor-2 and -4 on leukocytes from patients with sepsis. Shock 22:403-409

34. Escoll P, del Fresno C, García L, Vallés G, Lendínez MJ, Arnalich F, López-Collazo E (2003) Rapid up-regulation of IRAK-M expression following a second endotoxin challenge in human monocytes and in monocytes isolated from septic patients. Biochem Biophys Res Commun 311:465-472

35. Calvano JE, Agnese DM, Um JY, Goshima M, Singhal R, Coyle SM, Reddell MT, Kumar A, Calvano SE, Lowry SF (2003) Modulation of the lipopolysaccharide receptor complex (CD14, TLR4, MD-2) and toll-like receptor 2 in systemic inflammatory response syndrome-positive patients with and without infection: relationship to tolerance. Shock 20:415-419

36. Sfeir T, Saha DC, Astiz M, Rackow EC (2001) Role of interleukin-10 in monocyte hyporesponsiveness associated with septic shock. Crit Care Med 29:129-133

37. Heagy W, Hansen C, Nieman K, Cohen M, Richardson C, Rodriguez JL, West MA (2000) Impaired ex vivo lipopolysaccharide-stimulated whole blood tumor necrosis factor production may identify "septic" intensive care unit patients. Shock 14:271-276, discussion 276-7

38. Bergmann M, Gornikiewicz A, Sautner T, Waldmann E, Weber T, Mittlböck M, Roth E, Függer R (1999) Attenuation of catecholamine-induced immunosuppression in whole blood from patients with sepsis. Shock 12:421-427

39. Heagy W, Nieman K, Hansen C, Cohen M, Danielson D, West MA (2003) Lower levels of whole blood LPS-stimulated cytokine release are associated with poorer clinical outcomes in surgical ICU patients. Surg Infect (Larchmt) 4:171-180 
40. Flohé S, Lendemans S, Schade F-U, Kreuzfelder E, Waydhas C (2004) Influence of surgical intervention in the immune response of severely injured patients. Intensive Care Med 30:96-102

41. Buttenschoen K, Schneider ME, Utz K, Kornmann M, Beger HG, Carli Buttenschoen D (2009) Effect of major abdominal surgery on endotoxin release and expression of Toll-like receptors 2/4. Langenbecks Arch Surg 394:293-302

42. Kawasaki T, Ogata M, Kawasaki C, Tomihisa T, Okamoto K, Shigematsu A (2001) Surgical stress induces endotoxin hyporesponsiveness and an early decrease of monocyte mCD14 and HLA-DR expression during surgery. Anesth Analg 92:1322-1326

43. Roger P-M, Hyvernat H, Ticchioni M, Kumar G, Dellamonica J, Bernardin G (2012) The early phase of human sepsis is characterized by a combination of apoptosis and proliferation of T cells. J Crit Care 27:384-393

44. Zhang Y, Li J, Lou J, Zhou Y, Bo L, Zhu J, Zhu K, Wan X, Cai Z, Deng X (2011) Upregulation of programmed death-1 on T cells and programmed death ligand-1 on monocytes in septic shock patients. Crit Care 15:R70

45. Guignant C, Lepape A, Huang X, Kherouf H, Denis L, Poitevin F, Malcus C, Chéron A, Allaouchiche B, Gueyffier F, Ayala A, Monneret G, Venet F (2011) Programmed death-1 levels correlate with increased mortality, nosocomial infection and immune dysfunctions in septic shock patients. Crit Care 15:R99

46. Vaki I, Kranidioti H, Karagianni V, Spyridaki A, Kotsaki A, Routsi C, Giamarellos-Bourboulis EJ (2011) An early circulating factor in severe sepsis modulates apoptosis of monocytes and lymphocytes. J Leukoc Biol 89:343-349

47. Gogos C, Kotsaki A, Pelekanou A, Giannikopoulos G, Vaki I, Maravitsa P, Adamis S, Alexiou Z, Andrianopoulos G, Antonopoulou A, Athanassia S, Baziaka F, Charalambous A, Christodoulou S, Dimopoulou I, Floros I, Giannitsioti E, Gkanas P, loakeimidou A, Kanellakopoulou K, Karabela N, Karagianni V, Katsarolis I, Kontopithari G, Kopterides P, Koutelidakis I, Koutoukas P, Kranidioti H, Lignos M, Louis K et al (2010) Early alterations of the innate and adaptive immune statuses in sepsis according to the type of underlying infection. Crit Care 14:R96

48. Hoogerwerf JJ, van Zoelen MA, Wiersinga WJ, van't Veer C, de Vos AF, de Boer A, Schultz MJ, Hooibrink B, de Jonge $E$, van derPoll T (2010) Gene expression profiling of apoptosis regulators in patients with sepsis. J Innate Immun 2:461-468

49. Yousef AA, Suliman GA, Mabrouk MM (2010) The value of correlation of serum 20 s proteasome concentration and percentage of lymphocytic apoptosis in critically ill patients: a prospective observational study. Crit Care 14:R215

50. Turrel-Davin F, Guignant C, Lepape A, Mougin B, Monneret G, Venet F (2010) Upregulation of the pro-apoptotic genes BID and FAS in septic shock patients. Crit Care 14:R133

51. Pelekanou A, Tsangaris I, Kotsaki A, Karagianni V, Giamarellou H, Armaganidis A, Giamarellos-Bourboulis EJ (2009) Decrease of CD4-lymphocytes and apoptosis of CD14-monocytes are characteristic alterations in sepsis caused by ventilator-associated pneumonia: results from an observational study. Crit Care 13:R172

52. Delogu G, Famularo G, Tellan G, Marandola M, Antonucci A, Signore M, Marcellini S, Moretti S (2008) Lymphocyte apoptosis, caspase activation and inflammatory response in septic shock. Infection 36:485-487

53. Weber SU, Schewe J, Lehmann LE, Müller S, Book M, Klaschik S, Hoeft A, Stüber F (2008) Induction of Bim and Bid gene expression during accelerated apoptosis in severe sepsis. 12:1-10. Crit Care 12(5):R128

54. Roth G, Moser B, Krenn C, Brunner M, Haisjackl M, Almer G, Gerlitz S, Wolner E, Boltz-Nitulescu G, Ankersmit HJ (2003) Susceptibility to programmed cell death in T-lymphocytes from septic patients: a mechanism for lymphopenia and Th2 predominance. Biochem Biophys Res Commun 308:840-846

55. Le Tulzo Y, Pangault C, Gacouin A, Guilloux V, Tribut O, Amiot L, Tattevin P, Thomas R, Fauchet R, Drénou B (2002) Early circulating lymphocyte apoptosis in human septic shock is associated with poor outcome. Shock 18:487-494

56. Hotchkiss RS, Tinsley KW, Swanson PE, Schmieg RE, Hui JJ, Chang KC, Osborne DF, Freeman BD, Cobb JP, Buchman TG, Karl IE (2001) Sepsis-induced apoptosis causes progressive profound depletion of B and CD4+ T lymphocytes in humans. J Immunol 166:6952-6963

57. Hotchkiss RS, Swanson PE, Freeman BD, Tinsley KW, Cobb JP, Matuschak GM, Buchman TG, Karl IE (1999) Apoptotic cell death in patients with sepsis, shock, and multiple organ dysfunction. Crit Care Med 27:1230-1251

58. Bandyopadhyay G, Bankey PE, Miller-Graziano CL (2012) Trauma patients' elevated tumor necrosis related apoptosis inducing ligand (TRAIL) contributes to increased T cell apoptosis. Clin Immunol 145:44-54

59. Pellegrini JD, De AK, Kodys K, Puyana JC, Furse RK, Miller-Graziano C (2000) Relationships between T lymphocyte apoptosis and anergy following trauma. J Surg Res 88:200-206

60. Hotchkiss RS, Schmieg RE, Swanson PE, Freeman BD, Tinsley KW, Cobb JP, Karl IE, Buchman TG (2000) Rapid onset of intestinal epithelial and lymphocyte apoptotic cell death in patients with trauma and shock. Crit Care Med 28:3207-3217

61. Papadima A, Boutsikou M, Lagoudianakis EE, Kataki A, Konstadoulakis M, Georgiou L, Katergiannakis V, Manouras A (2009) Lymphocyte apoptosis after major abdominal surgery is not influenced by anesthetic technique: a comparative study of general anesthesia versus combined general and epidural analgesia. J Clin Anesth 21:414-421

62. Słotwiński R, Olszewski W, Słodkowski M, Lech G, Zaleska M, Kędziora S, Włuka A, Domaszewska A, Słotwińska S, Krasnodębski W, Wójcik Z (2011) Apoptosis in lymphocytes of pancreatic cancer patients: influence of preoperative enteral immunonutrition and extensive surgery. Arch Immunol Ther Exp (Warsz) 59:385-397

63. Delogu G, Famularo G, Moretti S, De Luca A, Tellan G, Antonucci A, Marandola M, Signore L (2001) Interleukin-10 and apoptotic death of circulating lymphocytes in surgical/anesthesia trauma. J Trauma 51:92-97

64. Delogu G, Moretti S, Antonucci A, Marcellini S, Masciangelo R, Famularo G, Signore L, De Simone C (2000) Apoptosis and surgical trauma: dysregulated expression of death and survival factors on peripheral lymphocytes. Arch Surg 135:1141-1147

65. Sasajima K, Inokuchi K, Onda M, Miyashita M, Okawa Kl, Matsutani T, Takubo K (1999) Detection of T cell apoptosis after major operations. Eur J Surg 165:1020-1023

66. Sugimoto M, Shimaoka M, Hosotsubo K, Tanigami H, Taenaka N, Kiyono H, Yoshiya I (1998) Up-regulation of Fas ligand (FasL) mRNA expression in peripheral blood mononuclear cells (PBMC) after major surgery. Clin Exp Immunol 112:120-125

67. Montecino-Rodriguez E, Berent-Maoz B, Dorshkind K (2013) Causes, consequences, and reversal of immune system aging. J Clin Invest 123:958-965 
68. Frink M, Pape H-C, van Griensven M, Krettek C, Chaudry IH, Hildebrand F (2007) Influence of sex and age on mods and cytokines after multiple injuries. Shock 27:151-156

69. Sakr Y, Elia C, Mascia L, Barberis B, Cardellino S, Livigni S, Fiore G, Filippini C, Ranieri V (2013) The influence of gender on the epidemiology of and outcome from severe sepsis. Crit Care 17:R50

70. Mayr FB, Yende S, Linde-Zwirble WT, Peck-Palmer OM, Barnato AE, Weissfeld LA, Angus DC (2010) Infection rate and acute organ dysfunction risk as explanations for racial differences in severe sepsis. JAMA 303:2495-2503

71. Fildes JE, Shaw SM, Yonan N, Williams SG (2009) The immune system and chronic heart failure: is the heart in control? J Am Coll Cardiol 53:1013-1020

72. Schreiber RD, Old L, Smyth MJ (2011) Cancer immunoediting: integrating immunity's roles in cancer suppression and promotion. Science 331:1565-1570

73. Bone RC, Balk RA, Cerra FB, Dellinger RP, Fein AM, Knaus WA, Schein RM, Sibbald WJ (1992) Definitions for sepsis and organ failure and guidelines for the use of innovative therapies in sepsis. The ACCP/SCCM Consensus Conference Committee. American College of Chest Physicians/Society of Critical Care Medicine. Chest 101:1644-1655

74. Levy MM, Fink MP, Marshall JC, Abraham E, Angus D, Cook D, Cohen J, Opal SM, Vincent J-L, Ramsay G (2003) 2001 SCCM/ESICM/ACCP/ATS/SIS International Sepsis Definitions Conference. Crit Care Med 31:1250-1256

75. Dellinger RP, Levy MM, Carlet JM, Bion J, Parker MM, Jaeschke R, Reinhart K, Angus DC, Brun-Buisson C, Beale R, Calandra T, Dhainaut J-F, Gerlach H, Harvey M, Marini JJ, Marshall J, Ranieri M, Ramsay G, Sevransky J, Thompson BT, Townsend S, Vender JS, Zimmerman JL, Vincent J-L (2008) Surviving Sepsis Campaign: international guidelines for management of severe sepsis and septic shock: 2008. Intensive Care Med 34:17-60

76. Zhang Q, Raoof M, Chen Y, Sumi Y, Sursal T, Junger W, Brohi K, Itagaki K, Hauser CJ (2010) Circulating mitochondrial DAMPs cause inflammatory responses to injury. Nature 464:104-107

77. Menu P, Vince JE (2011) The NLRP3 inflammasome in health and disease: the good, the bad and the ugly. Clin Exp Immunol 166:1-15

78. Vandenesch F, Lina G, Henry T (2012) Staphylococcus aureus hemolysins, bi-component leukocidins, and cytolytic peptides: a redundant arsenal of membrane-damaging virulence factors? Front Cell Infect Microbiol 2:12

79. Kanoh S, Rubin BK (2010) Mechanisms of action and clinical application of macrolides as immunomodulatory medications. Clin Microbiol Rev 23:590-615

80. Mikawa K, Akamatsu H, Maekawa N, Nishina K, Niwa Y (1998) Propofol inhibits human neutrophil. Anesth Analg 87:695-700

81. Vallejo R, de Leon-Casasola O, Benyamin R (2004) Opioid therapy and immunosuppression: a review. Am J Ther 11:354-365

82. Lawson EH, Louie R, Zingmond DS, Brook RH, Hall BL, Han L, Rapp M, Ko CY (2012) A comparison of clinical registry versus administrative claims data for reporting of 30-day surgical complications. Ann Surg 256:973-981

83. Knaus WA, Draper EA, Wagner DP, Zimmerman JE (1985) APACHE II: a severity of disease classification system. Crit Care Med 13:818-829

84. Knaus WA, Wagner DP, Draper EA, Zimmerman JE, Bergner M, Bastos PG, Sirio CA, Murphy DJ, Lotring T, Damiano A (1991) The APACHE III prognostic system. Risk prediction of hospital mortality for critically ill hospitalized adults. Chest 100:1619-1636

85. ASA Physical Status Classification System. http://www.asahq.org/For-Members/Clinical-Information/ASA-PhysicalStatus-Classification-System.aspx. Accessed 27 Jun 2013

86. Garner JS, Jarvis WR, Emori TG, Horan TC, Hughes JM (1989) CDC definitions for nosocomial infections, 1988. Am Rev Respir Dis 139:1058-1059

87. Baker SP, O'Neill B, Haddon W, Long WB (1974) The injury severity score: a method for describing patients with multiple injuries and evaluating emergency care. J Trauma 14:187-196

doi:10.1186/2197-425X-2-6

Cite this article as: Cain et al: Uncontrolled sepsis: a systematic review of translational immunology studies in intensive care medicine. Intensive Care Medicine Experimental 2014 2:6.

\section{Submit your manuscript to a SpringerOpen ${ }^{\circ}$ journal and benefit from:}

- Convenient online submission

- Rigorous peer review

- Immediate publication on acceptance

- Open access: articles freely available online

- High visibility within the field

- Retaining the copyright to your article

Submit your next manuscript at $\gg$ springeropen.com 